TRANSACTIONS OF THE

AMERICAN MATHEMATICAL SOCIETY

Volume 365, Number 2, February 2013, Pages 671-719

S 0002-9947(2012)05570-X

Article electronically published on July 19, 2012

\title{
COCYCLES AND CONTINUITY
}

\author{
HOWARD BECKER
}

\begin{abstract}
The topic of this paper is the Mackey Cocycle Theorem: every Borel almost cocycle is equivalent to a Borel strict cocycle. This is a theorem about locally compact groups which is not true for arbitrary Polish groups. We discuss the theorem, the open question of whether the theorem generalizes to some nonlocally compact Polish groups, the generalization to non-Borel cocycles, and other subjects associated with the theorem. Traditionally, the subject of cocycles and related matters has been considered in the context of standard Borel $G$-spaces. It is now known that a standard Borel $G$-space has a topological realization as a Polish $G$-space. This makes it possible to consider the subject from a topological point of view. The main theorem of this paper is that the conclusion of the Mackey Cocycle Theorem is equivalent to continuity properties of the almost cocycle. Even in the locally compact case, this continuity is a new result.
\end{abstract}

\section{BACKGRound AND STATEMENT OF THEOREMS}

The material in Section 1 of this paper is of three types. One is the statement of a large number of theorems, some old - such as the Mackey Cocycle Theorem (Theorem 1.3.11) - and some new - such as the paper's Main Theorem (Theorem 1.4.7). Very little is proved in Section 1; the proofs of the new theorems are all postponed until Section 2. This will, hopefully, make Section 1 easy reading.

Section 1 is semi-expository, and a second type of material is a general discussion of the following: the Mackey Cocycle Theorem, the open question of whether the theorem generalizes to some nonlocally compact Polish groups, the origin of this question, and various related matters. References are given for a more thorough discussion. This is a paper in the intersection of descriptive set theory and infinite dimensional group representation theory. It contains the background material necessary to make the paper accessible to mathematicians from both fields.

The last type of material in Section 1 is the usual preliminaries needed to understand the first two types: definitions, notation, and statements of relevant known theorems. The preliminary material which is needed for the proofs, but not needed for Section 1, is postponed until Section 2. We wish to warn the reader that notation, terminology and conventions vary widely in the literature. Even the sources cited in this paper often have notation, terminology and conventions different from - and sometimes incompatible with - that of this paper.

1.1. Groups and actions. We give a brief introduction to the subject of Polish groups and their actions. We refer the reader to Becker and Kechris [3] for information on this subject.

Received by the editors March 21, 2010 and, in revised form, February 16, 2011.

2010 Mathematics Subject Classification. Primary 03E15, 22A25, 28D15, 37A20, 43A65.

(C)2012 American Mathematical Society

Reverts to public domain 28 years from publication 
A. Polish groups. A Polish space is a separable, completely metrizable topological space. If $\langle X, t\rangle$ is a Polish space, we call $t$ a Polish topology on $X$.

A topology on a group is a group topology if the group operation and inversion are continuous. A topological group is a group together with its group topology. A Polish group is a topological group whose topology is Polish.

The branch of mathematics called "topological groups" has been concerned almost exclusively with locally compact groups. (A locally compact group is Polish iff it is second countable.) More recently, there has been an interest in the nonlocally compact case, an interest that emanates from diverse sources: for this author, from logic (Becker 2 and Becker and Kechris 3) ; for some, from physics (see §1.3.F, below); and for others, from operator algebras, infinite dimensional representation theory, ergodic theory, and elsewhere. There is an algorithm for producing open problems in this subject: take a known theorem about locally compact groups and ask whether - or to what extent - it is true in the nonlocally compact case. This paper is, in part, research of the above form; it was motivated entirely by the question of whether the Mackey Cocycle Theorem (Theorem 1.3.11), known to hold for locally compact groups, also holds for some nonlocally compact Polish groups. Although that question was the motivation, this paper does not provide an answer to the question. This paper is mostly concerned with the connection between the conclusion of the Mackey Cocycle Theorem and continuity properties of cocycles. While parts of this paper are known or vacuous in the locally compact case, not all of it is. For example, Corollary 1.4.8 is a new result about locally compact groups. Since this paper is about nonlocally compact groups, most of the usual subject matter of "topological groups" is irrelevant, and the reader who is ignorant of this subject is at no disadvantage.

Two examples of nonlocally compact Polish groups that will be mentioned in the sequel are the following. First, the unitary group, i.e., the group of unitary operators on a separable, infinite-dimensional Hilbert space, with the weak operator topology (equivalently, with the strong operator topology). Second, $S_{\infty}$, the group of permutations of $\mathbb{N}$, topologized as a $\left(G_{\delta}\right.$, hence Polish) subspace of $\mathbb{N}^{\mathbb{N}}$.

Definition 1.1.1. A nice group is a topological group of the form $C(K, G)$, where $K$ is a compact Polish space and $G$ is a locally compact Polish group; that is, it is the group of continuous functions from $K$ into $G$, with pointwise operations and the uniform topology.

Nice groups are Polish. Except in trivial cases, they are not locally compact. Nice groups have many properties not shared by arbitrary Polish groups, e.g., they admit complete left-invariant metrics, while Polish groups such as $S_{\infty}$ do not. (See [2] for information on groups admitting such metrics.)

B. Standard Borel $G$-spaces. A Borel space is a nonempty set, together with a $\sigma$-algebra of its subsets, called the Borel sets. Every topological space gives us a Borel space: a set is Borel if it is in the $\sigma$-algebra generated by the open sets. One can form products and subspaces of Borel spaces in the obvious way. A function between two Borel spaces is Borel-measurable (or simply Borel) if the preimage of a Borel set is Borel. Two Borel spaces are Borel-isomorphic if there exists a bijection, $f$, between them such that $f$ and $f^{-1}$ are both Borel-measurable.

A standard Borel space is a Borel space which is Borel-isomorphic to a Polish space. Of course, two different Polish topologies on $X$ may have the same Borel 
structure, that is, produce the same Borel sets. It is often convenient to work with standard Borel spaces rather than Polish spaces: first, because the topology may be irrelevant; and second, because the Borel structure may be very natural, while any topological realization is unnatural. The reader should consult Kechris 7, Chapter II] for information on Borel spaces and related topics.

Throughout this paper, a group, $G$, acts on a set, $X$. The action, $a$, is a function, $a: G \times X \rightarrow X$. $G$ acts on the left, and we use the dot notation accordingly:

$$
a:(g, x) \mapsto g \cdot x .
$$

(The dot is in contrast to multiplication in the group, which has no dot.) A $G$-space is a pair $(X, a)$ consisting of the set $X$ and the action, $a$, by $G$, on $X$. We generally suppress the $a$, and refer to "the $G$-space $X$ ". Since there is only one action on $X$ under consideration, this will cause no confusion.

Given a $G$-space $X$, a subset $Y$ of $X$ is invariant if for all $x \in Y$, for all $g \in$ $G, g \cdot x \in Y$. The action partitions $X$ into orbits, which are the smallest nonempty invariant sets. The action is transitive if it has only one orbit. Given a point $x \in X$, the stabilizer subgroup of $G$ at $x$, denoted $G_{x}$, is $\{g \in G: g \cdot x=x\}$.

Definition 1.1.2. Let $G$ be a Polish group, and let $X$ be a $G$-space under the action $a: G \times X \rightarrow X$.

(a) $X$ is called a standard Borel $G$-space if $X$ is a standard Borel space and a is Borel-measurable.

(b) $X$ is called a Polish $G$-space if $X$ is a Polish space and $a$ is continuous.

Of course, a Polish $G$-space is a standard Borel $G$-space.

Definition 1.1.3. Let $G$ be a Polish group, and let $X$ be a standard Borel $G$-space under the action $a: G \times X \rightarrow X$. A compatible topology for $X$ is a topology, $t$, satisfying the following three properties.

(a) $t$ is a Polish topology on $X$.

(b) The Borel structure of the topological space $\langle X, t\rangle$ is the original Borel structure of the standard Borel space $X$.

(c) The action $a$ is continuous with respect to $t$ (and the Polish topology on $G)$.

That is, a compatible topology turns a standard Borel $G$-space into a Polish $G$-space.

Theorem 1.1.4 (Becker and Kechris [3, 5.2.1]). Let $G$ be a Polish group, and let $X$ be a standard Borel $G$-space. There exists a compatible topology for $X$.

If $X$ is a transitive $G$-space, then by a theorem of Effros (see [4, page 251]), the compatible topology is unique. In general, it is not unique. In $\S 2.1$.D, we consider which sets can be made open in a compatible topology. We note here that given a countable collection of compatible topologies, there is another compatible topology finer than each of them (where finer means larger, i.e., with more open sets).

In this paper, $G$ and the standard Borel $G$-space $X$ are fixed. But we consider many different compatible topologies for $X$. 
1.2. Measures. We consider measures defined on standard Borel spaces. In this situation, the $\sigma$-algebra of measurable sets is the Borel sets.

\section{A. Measured Borel $G$-spaces.}

Definition 1.2.1. Let $X$ be a standard Borel $G$-space. Let $\mu$ be a probability measure on $X$. The measure $\mu$ is said to be quasi-invariant (with respect to the action) when for all Borel $A \subset X$ and for all $g \in G$,

$$
\mu(A)=0 \text { iff } \mu(g \cdot A)=0 .
$$

Any standard Borel $G$-space $X$ gives us an action by $G$ on the set of Borel probability measures on $X$. Specifically, $g \cdot \mu$ is the following measure: for all Borel $A \subset X$,

$$
(g \cdot \mu)(A)=\mu\left(g^{-1} \cdot A\right) .
$$

If $\mu$ is quasi-invariant, the measures $\mu$ and $g \cdot \mu$ are mutually absolutely continuous.

Definition 1.2.3. A measured Borel (respectively, Polish) G-space is a pair $(X, \mu)$, where $X$ is a standard Borel (respectively, Polish) $G$-space and $\mu$ is a probability measure on $X$ which is quasi-invariant.

Here is the simplest example. Let $G$ be a closed subgroup of a locally compact group, $H$. Let $X$ be the coset space $H / G$; the natural action of $G$ on $X$ turns $X$ into a standard Borel $G$-space. Let $\nu$ be Haar measure on $H$. This induces a measure, $\hat{\nu}$, on $X$ as follows: any Borel set $A \subset H / G$ can be regarded as a Borel subset of $H$ (the union of the cosets); set $\hat{\nu}(A)=\nu(A)$. Let $\mu$ be a probability measure such that $\mu$ and $\hat{\nu}$ are mutually absolutely continuous. Then $(X, \mu)$ is a measured Borel $G$-space.

\section{B. Suitable measures.}

Definition 1.2.4. Let $G$ be a Polish group, and let $(X, \mu)$ be a measured Borel $G$-space.

(a) A Borel set $Q \subset X$ is called almost invariant if for all $g \in G$, for $\mu$-a.e. $x \in Q, g \cdot x \in Q$.

(b) $(X, \mu)$ is called weakly suitable if for every almost invariant Borel set $Q \subset X$, there exists an invariant Borel set $Q^{\prime} \subset X$ such that $\mu\left(Q \triangle Q^{\prime}\right)=0$.

We also call $\mu$ a weakly suitable measure on the $G$-space $X$.

Theorem 1.2.9 below gives us many examples of weakly suitable $(X, \mu)$. We now take up a harder problem: Does there exist an $(X, \mu)$ which is not weakly suitable?

Definition 1.2.5. $X_{0}$ denotes the following Polish $S_{\infty}$-space. The points in $X_{0}$ are

$$
\begin{aligned}
&\left\{x \in 2^{\mathbb{N}}: \text { for infinitely many } n \in \mathbb{N}, x(n)=0\right. \\
&\text { and for infinitely many } n \in \mathbb{N}, x(n)=1\} .
\end{aligned}
$$

This is topologized as a subspace of $2^{\mathbb{N}}$. $S_{\infty}$, the group of permutations of $\mathbb{N}$, acts on $X_{0}$ by permuting coordinates: $(g \cdot x)(n)=x\left(g^{-1}(n)\right)$.

Theorem 1.2.6 (Kolmogorov-see [17]). There exists a measure $\mu$ on $X_{0}$ such that $\left(X_{0}, \mu\right)$ is a measured Polish $S_{\infty}$-space but $\left(X_{0}, \mu\right)$ is not weakly suitable. 
Definition 1.2.7. Let $G$ be a Polish group, and let $(X, \mu)$ be a measured Borel $G$-space. $(X, \mu)$ is called suitable if for all Borel $A, B \subset X$, one of the following two cases holds:

(1) for any open neighborhood $M$ of the group identity, there exists a $g \in M$ such that $\mu(\{x \in A: g \cdot x \in B\})>0$;

(2) there exist Borel sets $A^{\prime} \subset A$ and $B^{\prime} \subset B$ with $\mu\left(A \backslash A^{\prime}\right)=\mu\left(B \backslash B^{\prime}\right)=0$ and there exists an open neighborhood $N$ of the group identity such that $\left(N \cdot A^{\prime}\right) \cap B^{\prime}=\emptyset$.

We also called $\mu$ a suitable measure on the $G$-space $X$. The terminology of Definitions 1.2.4(b) and 1.2.7 is justified by the following theorem.

Theorem 1.2.8. Let $G$ be a Polish group, and let $(X, \mu)$ be a measured Borel $G$-space. If $(X, \mu)$ is suitable, then it is weakly suitable.

The author does not know whether the converse of Theorem 1.2.8 is true.

Theorem 1.2.8 will be proved in $\S 2.2$, as will the next theorem.

Theorem 1.2.9. Let $G$ be a locally compact Polish group. Every measured Borel $G$-space is suitable.

\section{Fulfillments.}

Definition 1.2.10. Let $G$ be a Polish group, let $(X, \mu)$ be a measured Borel $G$ space, and let $B$ be a Borel subset of $X$.

(a) A fulfillment of $B$ is an ordered pair $(\bar{E}, \bar{g})$, where $\bar{E}=E_{0}, E_{1}, E_{2}, \ldots$ is a sequence of Borel subsets of $X$ and $\bar{g}=g_{0}, g_{1}, g_{2}, \ldots$ is a sequence of elements of $G$, such that if $\widetilde{E}=\bigcup_{i} E_{i}$, then the three conditions below are satisfied.

(i) For all $i, j \in \mathbb{N}$, if $i \neq j$, then $E_{i} \cap E_{j}=\emptyset$.

(ii) For all $i \in \mathbb{N}, g_{i} \cdot E_{i} \subset B$.

(iii) $\mu((G \cdot B) \backslash \widetilde{E})=0$.

(b) Given $B$ and a fulfillment $(\bar{E}, \bar{g})$ of $B$, as above, the fulfillment function is the function $f: \widetilde{E} \rightarrow G$ defined as: $f(x)=g_{i}$, if $x \in E_{i}$.

In general, there will not be any fulfillment. For example, suppose that $\mu$ is a continuous measure, $X$ has exactly two orbits, each of positive measure, and $A \subset X$ consists of one orbit plus one point from the second orbit. It is easy to see that there does not exist a fulfillment of $A$.

But by removing one point, we obtain a Borel $B \subset A$ such that $\mu(B)=\mu(A)$ and such that (trivially) there is a fulfillment of $B$. That is the most we can hope for. And if $(X, \mu)$ is weakly suitable, we get it.

Theorem 1.2.11. Let $G$ be a Polish group, and let $(X, \mu)$ be a weakly suitable measured Borel $G$-space. Let $A \subset X$ be Borel. There exists a Borel set $B \subset A$ with $\mu(B)=\mu(A)$, and there exists a fulfilment of $B$.

Theorem 1.2.11 will be proved in $\S 2.2$. This theorem is not actually used in proving the Main Theorem of this paper (Theorem 1.4.7). However, the following theorem, which will be proved in $\S 2.3$, is needed for the Main Theorem.

Theorem 1.2.12. Let $G$ be a Polish group, and let $(X, \mu)$ be a suitable measured Borel $G$-space. Let $A \subset X$ be Borel. There exists a compatible topology, $t$, for $X$, 
there exists a Borel set $B \subset A$ with $\mu(B)=\mu(A)$, and there exists a fulfillment $(\bar{E}, \bar{g})$ of $B$ whose fulfillment function $f: \widetilde{E} \rightarrow G$ is continuous with respect to $t$.

The primary reason - perhaps the only reason - that suitable measures and fulfillments are of interest is their connection with cocycles. Nevertheless, cocycles are not actually involved in Theorem 1.2.12, and it is not even necessary to know the definition of cocycle to read the proof of Theorem 1.2.12. Although Theorem 1.2.12 might be regarded as just a lemma in the proof of the Main Theorem, it happens to be where most of the work is: the proof of Theorem 1.2.12 in $\S 2.3$ is longer and more complicated than any other proof in this paper. It is also the only place in this paper in which an understanding of compatible topologies is necessary.

1.3. Cocycles. The topic of this paper derives from group representation theory, a branch of pure mathematics which has a connection to physics. Cocycles provide a method of constructing representations. In this section, we give the information about cocycles which is needed to read the rest of the paper, but not much more. The reader is referred to Fabec [4, Chapters IV \& V] or Varadarajan [15, Chapters $\mathrm{V} \& \mathrm{VI}$ ] for additional information on cocycles, as well as for the background in representation theory.

\section{A. Strict cocycles.}

Definition 1.3.1. Let $G$ and $H$ be Polish groups, and let $X$ be a standard Borel $G$-space. A strict cocycle on $X$ with values in $H$ is a function $\varphi: G \times X \rightarrow H$ such that for all $g_{1}, g_{2} \in G$ and for all $x \in X$,

$$
\varphi\left(g_{1} g_{2}, x\right)=\varphi\left(g_{1}, g_{2} \cdot x\right) \varphi\left(g_{2}, x\right) .
$$

The above equation is called the cocycle identity. $H$ is called the target group.

Example 1.3.2. Let $G$ and $H$ be Polish groups, let $X$ be a transitive Polish $G$ space, and fix a point $p \in X$. Consider the stabilizer subgroup, $G_{p}$, which is a closed subgroup of $G$. Let $\pi: G_{p} \rightarrow H$ be a continuous homomorphism. As the action is transitive, for every $x \in X$, there exists a $g \in G$ such that $g \cdot p=x$. Let $\sigma: X \rightarrow G$ be a choice function that selects such a $g$, i.e., for all $x \in X, \sigma(x) \cdot p=x$. Define $\varphi: G \times X \rightarrow H$ as follows:

$$
\varphi(g, x)=\pi\left((\sigma(g \cdot x))^{-1} g \sigma(x)\right) .
$$

It is easy to check that the argument of $\pi$ is in $G_{p}$, hence $\varphi$ is well defined, and that $\varphi$ satisfies the cocycle identity.

For an arbitrary $G$-space $X, \varphi: G \times X \rightarrow H$ will be a strict cocycle iff the restriction of $\varphi$ to each orbit is a strict cocycle.

Using the Axiom of Choice, one can produce some very strange strict cocycles. We wish to exclude these from consideration. We will be considering only "definable" strict cocycles. It is traditional to consider Borel strict cocycles, that is, Borel-measurable functions $\varphi$, and that is almost always the case in this paper. But we occasionally also consider other types of definable cocycles, e.g., $\boldsymbol{\Delta}_{n}^{1}$ strict cocycles. Our discussion of the non-Borel case is confined entirely to Remark 1.3.3, \$1.3.C, Remark 1.3.13, Theorem 1.4.9, (1.4.10), and Remark 1.4.11; the reader who is only interested in the Borel case may safely skip these parts of the paper. 
Remark 1.3.3. For larger classes of definable sets, such as the $\boldsymbol{\Delta}_{n}^{1}$ sets, most questions are independent of ZFC, the usual axioms of set theory. (See [7] or [11.) In particular, some of our results on non-Borel cocycles require additional set theoretic assumptions that we will make precise when needed. Fortunately, everything in this paper involving Borel cocycles is proved in ZFC - it is ordinary mathematics. Thus the reader who wishes to skip the non-Borel parts of this paper, listed above, need not be concerned with set theoretic issues.

To return to Example 1.3.2, the definition of $\varphi$ depends on the choice function $\sigma$. The selection can be done in a Borel way. The fact that $\sigma$ can be taken to be Borel is a restatement of the theorem that there exists a Borel selector for cosets (see [7, 12.17]). In this case, $\varphi$ will also be Borel-measurable. So Example 1.3.2 provides an example of a Borel strict cocycle.

\section{B. Almost cocycles.}

Definition 1.3.4. Let $G$ and $H$ be Polish groups, and let $(X, \mu)$ be a measured Borel $G$-space.

(a) An almost cocycle on the measured Borel $G$-space $(X, \mu)$ with values in $H$ is a function $\varphi: G \times X \rightarrow H$ such that for all $g_{1}, g_{2} \in G$ and for $\mu$-a.e. $x \in X$,

$$
\varphi\left(g_{1} g_{2}, x\right)=\varphi\left(g_{1}, g_{2} \cdot x\right) \varphi\left(g_{2}, x\right) .
$$

(b) Two almost cocycles, $\varphi$ and $\psi$, on $(X, \mu)$ with values in $H$, are called equivalent when for all $g \in G$, for $\mu$-a.e. $x \in X, \varphi(g, x)=\psi(g, x)$.

Again, we are only interested in the case of definable almost cocycles, and usually of Borel almost cocycles.

A strict cocycle is an almost cocycle. If $\varphi$ is a strict cocycle and $\psi$ is equivalent to $\varphi$, then $\psi$ is also an almost cocycle. One might ask, Are these the only almost cocycles? That is, does there exist an almost cocycle which is not equivalent to a strict cocycle? That question is the central topic of this paper.

Example 1.3.5. Let $\mathbb{R}^{*}$ denote the multiplicative group of the positive real numbers. For any Polish group, $G$, and any measured Borel $G$-space, $(X, \mu)$, there is an equivalence class of Borel almost cocycles on $(X, \mu)$ with values in $\mathbb{R}^{*}$, which is called the Radon-Nikodym Cocycle, and which we now describe. If $\nu$ is a measure on $X$ which is absolutely continuous with respect to $\mu$, then there exists an $L_{1}$ function, $f$, such that for all Borel $A \subset X$,

$$
\nu(A)=\int_{A} f d \mu
$$

that function, $f$, is called the Radon-Nikodým derivative, and denoted $d \nu / d \mu$. Recall from (1.2.2) that for every $g \in G$, there exists a measure $g \cdot \mu$ that is absolutely continuous with respect to $\mu$. So we have a function $\varphi: G \times X \rightarrow \mathbb{R}^{*}$ such that

$$
\varphi(g, x)=\left[d\left(g^{-1} \cdot \mu\right) / d \mu\right](x) .
$$

That $\varphi$ is not unique, since "the" Radon-Nikodým derivative, $f$, is defined only up to almost everywhere equality. So for each $g \in G$, we must choose a representative of the $L_{1}$ function, and this choice function then yields $\varphi$. It is possible to do the choosing in a Borel way, in which case $\varphi$ is a Borel-measurable function. The cocycle identity is a consequence of the chain rule for Radon-Nikodým derivatives. 
But the chain rule for Radon-Nikodým derivatives only holds almost everywhere; so $\varphi$ is an almost cocycle. It is easy to see that two different choice functions give two equivalent almost cocycles. So we have an equivalence class of Borel almost cocycles. There is no obvious reason why this equivalence class should contain a strict cocycle. If, indeed, it does, that is a nontrivial theorem.

Definition 1.3.6. Let $G$ and $H$ be Polish groups, let $(X, \mu)$ be a measured Borel $G$-space, and let $\varphi$ and $\psi$ be two Borel-measurable almost cocycles on $(X, \mu)$ with values in $H$. We say that $\varphi$ is cohomologous to $\psi$ if there exists a Borel-measurable function $F: X \rightarrow H$ such that for all $g \in G$, for $\mu$-a.e. $x \in X$,

$$
\varphi(g, x)=F(g \cdot x) \psi(g, x)[F(x)]^{-1} .
$$

This defines an equivalence relation on the almost cocycles from $(X, \mu)$ into $H$. That equivalence relation is called cohomology. What we defined above is "almost cohomology"; one could define "strict cohomology", but we do not need that concept now.

Proposition 1.3.7. Let $G$ and $H$ be Polish groups, let $(X, \mu)$ be a measured Borel $G$-space, and let $\varphi$ and $\psi$ be Borel almost cocycles on $(X, \mu)$ with values in $H$.

(a) If $\varphi$ and $\psi$ are equivalent, then they are cohomologous.

(b) If $\varphi$ and $\psi$ are cohomologous and $\varphi$ is equivalent to a Borel strict cocycle, then so is $\psi$.

What we here call cohomology is sometimes called "equivalence". But in this paper, the term equivalence always has the meaning of Definition 1.3.4(b).

Cohomologous almost cocycles are often thought of as being the same.

A specific example of cohomology comes from the construction of Example 1.3.2, given a quasi-invariant measure, $\mu$, on $X$. The strict cocycle, $\varphi$, defined in Example 1.3.2 depends on a choice function $\sigma: X \rightarrow G$. Two different choice functions will yield cohomologous almost cocycles, but will not yield equivalent almost cocycles.

C. The non-Borel case. The topic of this paper can best be described as a partial answer to the following two vague questions.

Question 1. When is a Borel almost cocycle equivalent to a Borel strict cocycle?

Question 2. When is a strict cocycle equivalent to a Borel strict cocycle?

The purpose of Question 2 is to divide Question 1 into two parts. Suppose we have a Borel almost cocycle, $\varphi$, which is not equivalent to a Borel strict cocycle. There are two ways that this could happen: it may be that $\varphi$ is equivalent to a strict cocycle, but not to a Borel strict cocycle; or it may be that $\varphi$ is not equivalent to any strict cocycle at all. We wish to separate the descriptive set theory from the algebra/measure theory, and Question 2 is the most convenient way to do the separating.

In $\S \S 1.3 . \mathrm{D}$ and 1.3.E we are concerned with Question 1: positive results in $\S 1.3 . \mathrm{D}$; negative results in $\S 1.3$.E. The Main Theorem (Theorem 1.4.7) is also concerned with Question 1. The rest of $\S 1.3 . \mathrm{C}$ is about Question 2. For unexplained terminology in Definition 1.3.8 and the paragraph following, see [7.

Definition 1.3.8. Let $\mathcal{M}$ be a collection of pointsets (in all standard Borel spaces) such that every Borel set is in $\mathcal{M}$. $\mathcal{M}$ is called a useful pointclass if it satisfies the following three conditions. 
(a) All $\mathcal{M}$ sets are universally measurable, and all $\mathcal{M}$ subsets of a Polish space have the property of Baire.

(b) $\mathcal{M}$ is closed under complements, countable unions, measure quantifiers, and category quantifiers.

(c) The preimage of an $\mathcal{M}$ set under an $\mathcal{M}$-measurable function is an $\mathcal{M}$ set.

The smallest useful pointclass is the pointclass of Borel sets. The pointclass of $C$ sets is also useful. (Closure under category quantifiers is Vaught [16, 1.6]; a similar proof works for measure quantifiers.) For any $n \geq 1$, if $\boldsymbol{\Delta}_{n}^{1}$ sets are measurable and have the Baire property, then the pointclass of $\boldsymbol{\Delta}_{n}^{1}$ sets is useful. (For $n \geq 2$, it is not provable in ZFC that $\boldsymbol{\Delta}_{n}^{1}$ sets are measurable or have the Baire property; see [11, Chapter 5].)

Here is how the non-Borel case is handled in this paper: we do not do any serious proving of anything, we merely point to a Borel theorem and assert that the proof given for the pointclass of Borel sets actually works for any useful pointclass.

As is well known, if $\mathcal{M}$ sets are measurable (hence if the pointclass of $\mathcal{M}$ sets is useful), any $\mathcal{M}$-measurable function $f:[0,1] \rightarrow \mathbb{R}$ is equal almost everywhere to a Borel function. So if we only care about functions up to almost everywhere equality, there is no reason to ever consider anything other than Borel functions. Theorem 1.3.9, below, is a similar result for almost cocycles rather than real functions.

Theorem 1.3.9. Let $\mathcal{M}$ be a useful pointclass. Let $G$ and $H$ be Polish groups, let $(X, \mu)$ be a measured Borel $G$-space, and let $\varphi: G \times X \rightarrow H$ be an almost cocycle such that $\varphi$ is an $\mathcal{M}$-measurable function. There exists a Borel-measurable almost cocycle $\psi: G \times X \rightarrow H$ such that $\psi$ is equivalent to $\varphi$.

Proof. Fix $\varphi$. For $g \in G$, let $\varphi_{g}: X \rightarrow H$ be the function $\varphi_{g}(x)=\varphi(g, x)$. Let $d$ be a right-invariant metric on $H$ with finite diameter.

Claim. For any sequence $g_{n}$ of elements of $G$ and for any $g \in G$, if $g_{n}$ converges to $g$, then $\varphi_{g_{n}}$ converges to $\varphi_{g}$ in measure (with respect to $\mu$ and $d$ ).

In the case of Borel $\varphi$, this Claim is Lemma 2.4.7, proved in $\S 2.4$ as part of the proof of the Main Theorem. Since $\mathcal{M}$ is useful, the proof of Lemma 2.4.7, given in this paper for Borel-measurable $\varphi$, also works for $\mathcal{M}$-measurable $\varphi$, thus giving us the Claim.

Let $D$ be a countable dense subgroup of $G$. Clearly, for every $g \in D$, we can find a Borel function $f_{g}: X \rightarrow H$ such that $f_{g}$ is equal to $\varphi_{g} \mu$-almost everywhere. Now for each $g \in G$ we can choose - in a Borel way - a sequence $g_{n}$ from $D$ such that $g_{n} \rightarrow g$, and then construct-in a Borel way - a function $\psi_{g}: X \rightarrow H$ such that $f_{g_{n}}$ converges to $\psi_{g}$ in measure. We define $\psi: G \times X \rightarrow H$ by $\psi(g, x)=\psi_{g}(x)$. Then $\psi$ is Borel and equivalent to $\varphi$.

So if we only care about almost cocycles up to equivalence, there is no reason to ever consider anything other than Borel almost cocycles. But that is for almost cocycles, where one is allowed to ignore sets of measure 0 . The author does not know if this is also the case for strict cocycles. But if the measure is suitable, this is, indeed, the case.

Theorem 1.3.10. Let $\mathcal{M}$ be a useful pointclass. Let $G$ and $H$ be Polish groups, let $(X, \mu)$ be a suitable measured Borel $G$-space, and let $\varphi: G \times X \rightarrow H$ be a strict cocycle such that $\varphi$ is an $\mathcal{M}$-measurable function. There exists a Borel-measurable strict cocycle $\psi: G \times X \rightarrow H$ such that $\psi$ is equivalent to $\varphi$. 
A proof of Theorem 1.3.10 will be given in $\S 1.4 . \mathrm{C}$, after stating the Main Theorem of this paper.

\section{The Mackey Cocycle Theorem.}

Theorem 1.3.11 (Mackey Cocycle Theorem). Let $G$ be a locally compact Polish group, and let $H$ be an arbitrary Polish group. Let $(X, \mu)$ be a measured Borel $G$-space. For every Borel-measurable almost cocycle $\psi$ on $(X, \mu)$ with values in $H$, there exists a Borel-measurable strict cocycle $\varphi$ on $X$ with values in $H$ such that $\varphi$ is equivalent to $\psi$.

For a proof of Theorem 1.3.11, see [4, page 153] or [15, 5.26].

As mentioned in $\S 1.1$.A, this paper was motivated by the question of whether Theorem 1.3.11 also holds for some nonlocally compact $G$. For reasons that will be given in $\S 1.3 . \mathrm{F}$, this question is of particular interest in the case of unitary cocycles, i.e., those for which the target group is the unitary group. Not much can be said in a positive direction. There exist Polish groups with the property that all unitary almost cocycles are trivial (Herer and Christensen [6]). Such groups vacuously satisfy Mackey's Theorem for unitary cocycles. Aside from that, the author does not believe that any nonlocally compact Polish groups are known to satisfy Mackey's Theorem for unitary cocycles.

Remark 1.3.12. A related result, also due to Mackey, and often combined with the Mackey Cocycle Theorem is this: every system of imprimitivity arises from an almost cocycle. We do not give any definitions here. We merely remark that this result is true for arbitrary Polish groups, $G$, and the usual proof for locally compact $G$ (see [4, page 205] or [15, 6.11]) actually works for arbitrary $G$. Thus, if the Mackey Cocycle Theorem holds for some $G$, so does the "Imprimitivity Theorem".

In the nonlocally compact case, some instances of the Mackey Cocycle Theorem and/or the Imprimitivity Theorem have been proved; see Fidaleo [5] and the references therein. But these theorems put hypotheses on the measured Borel $G$-space, not just on the group $G$.

The proof of Theorem 1.3.11 uses Haar measure on the locally compact group $G$. It is a theorem of Weil (see [4, page 147]) that for nonlocally compact groups, there does not exist a $\sigma$-finite left-invariant measure. Nevertheless, it is possible that a modified form of Mackey's proof may work for some nonlocally compact groups. We discuss this scenario in the next remark. This remark may cause the reader who believes the non-Borel case to be uninteresting to reconsider this belief.

Remark 1.3.13. The full strength of Haar measure is not needed for the proof of Theorem 1.3.11. What is needed is an ideal $\mathcal{I}$ (not necessarily a $\sigma$-ideal) on $G$ such that $\mathcal{I}$ is left-invariant, open sets are not in $\mathcal{I}$, and $\mathcal{I}$ has the following Fubini-type property: for all $g \in G$, for $\mu$-a.e. $x \in X$. implies that for $\mu$-a.e. $x \in X$, for $\mathcal{I}$-a.e. $g \in G \ldots$ In the locally compact case, $\mathcal{I}$ is, of course, the ideal of measure 0 sets. It is not known whether it is possible for a nonlocally compact group to admit such an ideal. While arbitrary ideals with the Fubini property have been studied (e.g., Recław and Zakrzewski [13]) and arbitrary ideals on groups have been studied (e.g., Balcerzak [1), apparently the two topics have never been considered together. If such an ideal exists on $G$, then Mackey's proof of Theorem 1.3.11 will work, and we will obtain a strict cocycle, $\varphi$, equivalent to the original Borel almost cocycle $\psi$. But $\varphi$ will not be a Borel strict cocycle. It will, however, be $C$-measurable. That 
is because the proof of Theorem 1.3.11 uses local compactness in one other way, besides the existence of Haar measure. Specifically, it uses the fact that, since $F_{\sigma}$ subsets of $G$ are $\sigma$-compact, a Borel set in $X \times G$ with $F_{\sigma}$ sections admits a Borel uniformization (see $[7,18.18]$ ). Without $\sigma$-compactness, we do not necessarily get a Borel uniformization, but we do get a uniformization by a $C$-measurable function (see [7, 18.1]). To get an equivalent Borel strict cocycle from the $C$-measurable strict cocycle, we apply Theorem 1.3.10. The hypothesis of Theorem 1.3.10 requires the measured Borel $G$-space to be suitable. Theorem 1.2.9 states that for locally compact $G$, every measured Borel $G$-space is suitable. But if $G$ admits an ideal $\mathcal{I}$, as above, then the proof of Theorem 1.2.9 given in $\S 2.2$ also works for $G$. Thus the existence of such an ideal on $G$ gives us the entire Mackey Cocycle Theorem for $G$.

E. Counterexamples. Suppose we have two Polish groups $G$ and $H$, a measured Borel $G$-space $(X, \mu)$ and two Borel strict cocycles, $\varphi_{1}$ and $\varphi_{2}$, on $(X, \mu)$ with values in $H$. Let $Q \subset X$ be a Borel set. We can define a new Borel-measurable function $\tilde{\varphi}: G \times X \rightarrow H$ as follows:

$$
\tilde{\varphi}(g, x)= \begin{cases}\varphi_{1}(g, x), & \text { if } x \in Q, \\ \varphi_{2}(g, x), & \text { if } x \notin Q .\end{cases}
$$

Obviously, if $Q$ is invariant, $\tilde{\varphi}$ will also be a strict cocycle.

Now suppose $Q$ is almost invariant (see Definition 1.2.4). Then $\tilde{\varphi}$ is an almost cocycle. If there exists an invariant Borel $Q^{\prime} \subset X$ such that $\mu\left(Q \triangle Q^{\prime}\right)=0$, then $\tilde{\varphi}$ will be equivalent to a strict cocycle - the strict cocycle defined piecewise via $Q^{\prime}$ rather than $Q$. So if $(X, \mu)$ is weakly suitable, then any almost cocycle defined piecewise, as above, will be equivalent to a strict cocycle. But in the nonweakly suitable case, it seems that an almost cocycle $\tilde{\varphi}$ defined piecewise will probably not be equivalent to a strict cocycle. And, in some concrete cases, we can prove it is not.

Specifically, consider the transitive Polish $S_{\infty}$-space $X_{0}$ of Definition 1.2.5. We define two strict cocycles on $X_{0}$ with values in $S_{\infty}$, both of them special cases of the construction in Example 1.3.2.

Definition 1.3.14. (a) For $x \in X_{0}$, let $M_{0}(x)=\{n \in \mathbb{N}: x(n)=0\}, M_{1}(x)=$ $\{n \in \mathbb{N}: x(n)=1\}$. Let $\sigma: X_{0} \rightarrow S_{\infty}$ be the following function. For $x \in X_{0}, \sigma(x)$ is the unique permutation, $g$, of $\mathbb{N}$ such that for all $m \in \mathbb{N}$ :

(i) $g(2 m)=m$ th element of $M_{0}(x)$;

(ii) $g(2 m+1)=m$ th element of $M_{1}(x)$.

(b)

(i) The trivial cocycle, denoted $\varphi_{t}$, is the constant function on $S_{\infty} \times X_{0}$, whose value is the group identity of $S_{\infty}$.

(ii) The interesting cocycle, denoted $\varphi_{i}$, is the following function from $S_{\infty} \times X_{0}$ into $S_{\infty}$ :

$$
\varphi_{i}(g, x)=(\sigma(g \cdot x))^{-1} g \sigma(x) .
$$

Clearly $\varphi_{t}$ and $\varphi_{i}$ are both Borel strict cocycles.

Theorem 1.3.15. Let $\mu$ be any quasi-invariant Borel probability measure on the Polish $S_{\infty}$-space $X_{0}$. Let $Q \subset X_{0}$ be any Borel set such that $0<\mu(Q)<1$ and such that $Q$ is $\mu$-almost invariant. Let $\tilde{\varphi}: S_{\infty} \times X_{0} \rightarrow S_{\infty}$ be the function

$$
\tilde{\varphi}(g, x)= \begin{cases}\varphi_{t}(g, x), & \text { if } x \in Q, \\ \varphi_{i}(g, x), & \text { if } x \notin Q .\end{cases}
$$


Then $\tilde{\varphi}$ is a Borel almost cocycle on the measured $S_{\infty}$-space $\left(X_{0}, \mu\right)$, but $\tilde{\varphi}$ is not equivalent (with respect to $\mu$ ) to any Borel strict cocycle on the $S_{\infty}$-space $X_{0}$.

Theorem 1.3.15 will be proved in $\S 2.7$. This theorem, together with Theorem 1.2.6, demonstrates that the Mackey Cocycle Theorem is not true for arbitrary Polish groups.

As previously mentioned, the author does not know if there are any measured Borel $G$-spaces which are weakly suitable but not suitable. But if there are any, it seems likely that a similar construction would yield a counterexample. The author believes that if the Mackey Cocycle Theorem is ever proved for some nonlocally compact $G$, it will be part of the proof - or will fall out of the proof - that any measured Borel $G$-space is suitable.

Although the above piecewise construction gives a counterexample, this type of counterexample begs the question, since the pathology is in the measured Borel $G$-space, not in the almost cocycle. We have still not addressed the question of whether bad cocycles can happen to good $G$-spaces.

That is, do suitable $G$-spaces always satisfy Mackey's Theorem? We now give our second counterexample: a suitable space that does not. The counterexample involves the Radon-Nikodým derivative and the Radon-Nikodým Cocycle (see Example 1.3.5).

Definition 1.3.16. Let $\mathbb{T}$ denote the unit circle, let $\delta$ denote the usual metric on $\mathbb{T}$, and let $\lambda$ denote normalized Lebesgue measure on $\mathbb{T}$.

(a) Let

$$
\begin{aligned}
G^{0}= & \{g: g \text { is an orientation-preserving homeomorphism from } \mathbb{T} \\
& \text { onto itself and for all Borel } B \subset \mathbb{T}, \lambda(B)=0 \text { iff } \lambda(g[B])=0\} .
\end{aligned}
$$

(b) Let $\delta^{1}, \delta^{2}$, and $\delta^{0}$ denote the following functions from $G^{0} \times G^{0}$ into the non-negative real numbers:

$$
\begin{aligned}
& \delta^{1}(g, h)=\sup \{\delta(g(x), h(x)): x \in \mathbb{T}\}, \\
& \delta^{2}(g, h)=\int\left|\frac{d(g \cdot \lambda)}{d \lambda}-\frac{d(h \cdot \lambda)}{d \lambda}\right| d \lambda, \\
& \delta^{0}(g, h)=\delta^{1}(g, h)+\delta^{2}(g, h) .
\end{aligned}
$$

(c) Let $t^{0}$ denote the topology on $G^{0}$ given by the metric $\delta^{0}$.

Clearly $G^{0}$, with the operation of composition, is a group. We consider the evaluation action of $G^{0}$ on $\mathbb{T}: g \cdot x=g(x)$. Obviously, $\lambda$ is quasi-invariant with respect to this action. It is also clear that the function $\delta^{0}$ actually defines an (incomplete) metric on $G^{0}$, hence $t^{0}$ is a topology on $G^{0}$.

Theorem 1.3.17. $\quad$ (a) $t^{0}$ is a group topology on $G^{0}$.

(b) $t^{0}$ is a Polish topology on $G^{0}$.

Proof. Fix a point $y \in \mathbb{T}$ and consider the stabilizer subgroup

$$
G_{y}^{0}=\left\{g \in G^{0}: g \cdot y=y\right\} .
$$

Then $G^{0}$ is the direct sum of $G_{y}^{0}$ and the group of rotations of $\mathbb{T}$. Via this direct sum decomposition, this theorem will follow from these two facts: that $t^{0}$ is a group topology on $G_{y}^{0}$, and that $t^{0}$ is a Polish topology on $G_{y}^{0}$. 
Both of these facts are proved in Solecki [14, 2.4]; however, that paper describes the topological group $G_{y}^{0}$ in a different way than it is described here. First, that paper considers increasing homeomorphisms from $[0,1]$ to $[0,1]$, rather than orientation-preserving homeomorphisms of $\mathbb{T}$ that fix $y$. Second, it defines the group as those functions $f$ such that both $f$ and $f^{-1}$ are absolutely continuous; but absolute continuity is equivalent to moving sets of measure 0 to sets of measure 0 (see [14, 2.5]). Third, it defines the metric via the ordinary derivative of a real-valued function of a real variable; but in this situation, the ordinary derivative is the same thing as the Radon-Nikodým derivative in our definition of $\delta^{2}$.

The action of $G^{0}$ on $\mathbb{T}$ is as nice as an action can be, both algebraically and topologically - a transitive action and a continuous action on a compact set. The next theorem states that it is also nice measure theoretically.

Theorem 1.3.18. $\lambda$ is a suitable measure on the Polish $G^{0}$-space $\mathbb{T}$.

Theorem 1.3.18 will be proved in $\S 2.7$. The next result, which is also proved in $\S 2.7$, demonstrates that suitability is not a sufficient condition to imply the Mackey Cocycle Theorem.

Theorem 1.3.19. Let $\varphi: G^{0} \times \mathbb{T} \rightarrow \mathbb{R}^{*}$ be the Radon-Nikodým Cocycle for the measured Polish $G^{0}$-space $(\mathbb{T}, \lambda)$. Then $\varphi$ is not equivalent to any Borel-measurable strict cycle.

The two counterexamples demonstrate that neither $S_{\infty}$ nor $G^{0}$ admits an ideal of the type discussed in Remark 1.3.13. (For $S_{\infty}$, this actually follows from Theorem 1.2.6.) It can be shown, using [2, 3.B.2], that neither of these two groups is topologically isomorphic to a nice group (see Definition 1.1.1).

Conjecture 1.3.20. All nice groups satisfy the Mackey Cocycle Theorem.

We conclude $\S 1.3$.E with a point that is relevant to $\S 1.3$.F. In both counterexamples, the target group is a closed subgroup of the unitary group. Therefore, in both counterexamples, the target group can be taken to be the unitary group.

F. Foundations of quantum mechanics. Much of the interest in this topic derives from the foundations of quantum mechanics. The Mackey Cocycle Theorem has physical meaning when $G$ is a group of physical symmetries and $H$ is the unitary group. We shall give a very brief explanation of this; for more details, the reader should consult Mackey [9] or Varadarajan [15].

A group of physical symmetries, $G$, acts in a manner which produces an equivalence relation on the observables. One would like to know what the equivalence classes of the observables are. These equivalence classes are in one-to-one correspondence with the cohomology classes of Borel almost cocycles on a measured Borel $G$-space with values in the unitary group; and that correspondence is well understood. (The observables can be more accurately described in terms of systems of imprimitivity; cf. Remark 1.3.12.) If the Mackey Cocycle Theorem is true for $G$, that means that the only observables are the ones we know about - there are no others. If the Mackey Cocycle Theorem is false for $G$, that means that there are new observables which have not yet been discovered, and furthermore, these new observables are pathological.

It is not known what the "groups of physical symmetries" are, in terms of a mathematically defined class of groups. According to some physical theories, there 
are nonlocally compact nice groups which are groups of physical symmetries, e.g., gauge groups. The fact that some nice groups are groups of physical symmetries means that Conjecture 1.3.20 has physical meaning.

Although the topic of this paper has some relevance to physics, this is a paper in pure mathematics. Physics is never mentioned in this paper outside of $§ 1.3 . F$.

\subsection{The Main Theorem.}

A. Continuity of almost cocycles. Traditionally, the subject of cocycles and related matters has been considered in the context of standard Borel $G$-spaces. That is the way it is presented in books such as Fabec [4] and Varadarajan [15], and that is how it has been presented in this paper. The author has no doubt that, in the past, standard Borel $G$-spaces were the correct context for studying this topic - but the author is not sure that it still is.

It is now known that standard Borel $G$-spaces have topological realizations as Polish $G$-spaces (Theorem 1.1.4). This makes it possible to consider the topic from a topological point of view. In this paper, we re-examine one aspect of this subject from a topological point of view; perhaps in the future, other aspects should also be re-examined. Specifically, we consider continuity properties of almost cocycles.

Definition 1.4.1. Let $G$ and $H$ be Polish groups, let $(X, \mu)$ be a measured Polish $G$-space, and let $\psi$ be a Borel-measurable almost cocycle on $(X, \mu)$ with values in $H$.

(a) The function $\psi: G \times X \rightarrow H$ is called essentially continuous if there exists a Borel set $S \subset G \times X$ satisfying the following two conditions.

(i) For all $g \in G$, for $\mu$-a.e. $x \in X,(g, x) \in S$.

(ii) $\psi\lceil S$ is continuous.

(b) The function $\psi: G \times X \rightarrow H$ is called nearly essentially continuous if for all $\epsilon>0$ there exists a compact $K \subset X$ such that $\mu(K)>1-\epsilon$ and there exists a Borel set $S \subset G \times X$ satisfying the following two conditions.

(i) For all $g \in G$, for $\mu$-a.e. $x \in X$, if both $x$ and $g \cdot x$ are in $K$, then $(g, x) \in S$.

(ii) $\psi\lceil S$ is continuous.

It is easy to see that essential continuity is a property of the equivalence class; that is, if $\varphi$ and $\psi$ are equivalent almost cocycles, the essential continuity of $\varphi$ implies that of $\psi$. Essential continuity does not respect the coarser equivalence relation of cohomology. The function $F: X \rightarrow H$ in the definition of cohomologous (see Definition 1.3.6) has the property that for all $\epsilon>0, F$ is continuous on a subset of $X$ of measure $1-\epsilon$; from this it easily follows that near essential continuity is a property of the cohomology class. Two weak versions of that fact are contained in the following proposition; part (b) is a consequence of Proposition 1.3.7(a).

Proposition 1.4.2. Let $G$ and $H$ be Polish groups, let $(X, \mu)$ be a measured Polish $G$-space, and let $\varphi$ and $\psi$ be two Borel-measurable almost cocycles on $(X, \mu)$ with values in $H$.

(a) If $\varphi$ is cohomologous to $\psi$ and $\varphi$ is essentially continuous, then $\psi$ is nearly essentially continuous.

(b) If $\varphi$ is equivalent to $\psi$ and $\varphi$ is nearly essentially continuous, then so is $\psi$.

Now, back to standard Borel $G$-spaces. We consider compatible topologies (see Definition 1.1.3) for these spaces. 
Definition 1.4.3. Let $G$ and $H$ be Polish groups, let $(X, \mu)$ be a measured Borel $G$-space, and let $\psi$ be a Borel-measurable almost cocycle on $(X, \mu)$ with values in $H$. We call $\psi$ quintessentially continuous (respectively, nearly quintessentially continuous) if there exists a compatible topology, $t$, for $X$ such that $\psi$ is essentially continuous (respectively, nearly essentially continuous) with respect to the Polish $G$-space $\langle X, t\rangle$.

Note that if a compatible topology, $t$, satisfies the above definition, so does any compatible topology which is finer than $t$.

B. Statement of the Main Theorem. The Main Theorem of this paper, Theorem 1.4.7, states that the conclusion of the Mackey Cocycle Theorem is equivalent to continuity properties of the almost cocycle. This theorem breaks into three parts, which we call Theorems A, B, and C.

Theorem 1.4.4 (Theorem A). Let $G$ and $H$ be Polish groups, let $(X, \mu)$ be a measured Polish $G$-space, and let $\psi$ be a Borel-measurable almost cocycle on $(X, \mu)$ with values in $H$. If $\psi$ is essentially continuous, then there exists a Borel-measurable strict cocycle $\varphi$ on $X$ with values in $H$ such that $\varphi$ is equivalent to $\psi$.

Theorem A will be proved in $\S 2.4$.

Theorem 1.4.5 (Theorem B). Let $G$ and $H$ be Polish groups, let $(X, \mu)$ be a measured Polish $G$-space, and let $\varphi$ be a Borel-measurable almost cocycle on $(X, \mu)$ with values in $H$. If $\varphi$ is a strict cocycle, then $\varphi$ is nearly essentially continuous.

Theorem B will be proved in $\S 2.5$.

Theorem 1.4.6 (Theorem C). Let $G$ and $H$ be Polish groups, and let $(X, \mu)$ be a measured Borel $G$-space such that $\mu$ is suitable. Let $\psi$ be a Borel-measurable almost cocycle on $(X, \mu)$ with values in $H$. If $\psi$ is nearly quintessentially continuous, then there exists a Borel-measurable quintessentially continuous almost cocycle $\varphi$ on $(X, \mu)$ with values in $H$ such that $\varphi$ is cohomologous to $\psi$.

Theorem $\mathrm{C}$ will be proved in $\S 2.6$.

Theorem C is false for "essential" rather than "quintessential"; that is, in going from a nearly essentially continuous $\psi$ to an essentially continuous $\varphi$, we must change the topology.

Combining Theorems A, B, and C with Theorem 1.1.4, Proposition 1.3.7(b), and Proposition 1.4.2(b), we obtain the following.

Theorem 1.4.7 (Main Theorem). Let $G$ and $H$ be Polish groups, and let $(X, \mu)$ be a measured Borel $G$-space such that $\mu$ is suitable. Let $\psi$ be a Borel-measurable almost cocycle on $(X, \mu)$ with values in $H$. The following are equivalent.

(a) $\psi$ is equivalent to a Borel-measurable strict cocycle.

(b) $\psi$ is nearly quintessentially continuous.

(c) $\psi$ is cohomologous to a Borel-measurable almost cocycle which is quintessentially continuous.

For transitive actions, $(a) \Longrightarrow(c)$ of Theorem 1.4.7 was known (at least for some groups $G$ ), since it was known exactly what the cohomology classes of strict cocycles are: they are all obtained by the construction of Example 1.3.2. In general, however, it is a new result. 
The $(c) \Longrightarrow(b)$ implication of Theorem 1.4.7 is Proposition 1.4.2(a); in other words, it is trivial.

Of course the suitability assumption is not needed in proving $(c) \Longrightarrow(a)$, $(a) \Longrightarrow(b)$, or $(c) \Longrightarrow(b)$. Suitability is needed in proving the $(b) \Longrightarrow(a)$ implication of the Main Theorem, and therefore, also in proving Theorem $\mathrm{C}$ (the $(b) \Longrightarrow(c)$ implication). The author does not know whether or not $(a) \Longrightarrow(c)$ holds in the absence of suitability.

The fact that $(b) \Longrightarrow(a)$ of the Main Theorem may fail in the absence of suitability is demonstrated by the first counterexample to the Mackey Cocycle Theorem (Definition 1.3.14 and Theorem 1.3.15). The function $\tilde{\varphi}$, defined piecewise from $\varphi_{t}$ and $\varphi_{i}$ in that counterexample, is nearly essentially continuous. This fact follows easily from Theorem B, applied to the strict cocycles $\varphi_{t}$ and $\varphi_{i}$.

C. Applications of the Main Theorem. We conclude Section 1 with three applications of the Main Theorem, as well as a brief mention of two possible future applications.

Corollary 1.4.8. Let $G$ be a locally compact Polish group, let $H$ be an arbitrary Polish group, and let $(X, \mu)$ be an arbitrary measured Borel $G$-space. Let $\psi$ be a Borel-measurable almost cocycle on $(X, \mu)$ with values in $H$. Then $\psi$ is nearly quintessentially continuous and $\psi$ is cohomologous to a Borel-measurable almost cocycle which is quintessentially continuous.

Proof. Theorems 1.2.9, 1.3.11, and 1.4.7.

As mentioned several times in this paper, it would be desirable to prove the Mackey Cocycle Theorem for some nonlocally compact groups, e.g., nice groups. The author hopes that some day the Main Theorem (Theorem 1.4.7) may be used for that purpose: prove $(a)$ by establishing $(b)$. At the present time this is nothing more than a hope.

What is more than a hope is the converse: proving that (a) fails by establishing that (b) fails. This is the method of proof used in the second counterexample to the Mackey Cocycle Theorem: Theorem 1.3.19 is proved by establishing that the Radon-Nikodým Cocycle is not nearly quintessentially continuous, and then citing the Main Theorem.

The author also hopes that the Main Theorem might give us a better understanding of what the strict cocycles (up to cohomology) are - even in the locally compact case. For simplicity, let us consider a measured Borel $G$-space $(X, \mu)$ which is ergodic, that is, every invariant Borel subset of $X$ has measure 0 or 1 . There are two kinds of ergodic measured Borel $G$-space: transitive and properly ergodic. In the case of transitive actions, we know exactly what the strict cocycles are: up to cohomology, they are all of the form discussed in Example 1.3.2. In the properly ergodic case, Mackey [8] developed a concept of virtual groups which gives an analog of this result for the properly ergodic case, that is, a theorem that up to cohomology, all strict cocycles are of a specific form. In spite of the importance of this result, it is fair to say that it is still difficult to understand what the strict cocycles are in the properly ergodic case.

Consider the simplest case of a properly ergodic measured Borel $G$-space $(X, \mu)$ : the case in which $G$ is a countable discrete group. Note that a strict cocycle on $(X, \mu)$ is determined by the behavior of a cohomologous almost cocycle on just one orbit! Part $(c)$ of the Main Theorem tells us that the cohomologous almost cocycle 
extends by continuity from that one orbit to a set of measure 1 . The author does not know what this means or if it means anything at all. But - expressing another hope for the future- perhaps it can help us to understand what the strict cocycles are.

There is one remaining loose end to tie up: after stating Theorem 1.3.10, we promised to give the proof later. We now keep the promise - it is the third application of the Main Theorem. Of course, this topic is meaningful only in the non-Borel case.

Theorem 1.4.9. Let $\mathcal{M}$ be a useful pointclass. Let $G$ and $H$ be Polish groups, let $(X, \mu)$ be a measured Polish $G$-space, and let $\varphi$ be an $\mathcal{M}$-measurable almost cocycle on $(X, \mu)$ with values in $H$. If $\varphi$ is a strict cocycle, then $\varphi$ is nearly essentially continuous.

Proof. In the case of Borel $\varphi$, this result is Theorem B (Theorem 1.4.5). Since $\mathcal{M}$ is useful, the proof of Theorem B given in this paper for Borel-measurable $\varphi$ also works for $\mathcal{M}$-measurable $\varphi$.

\section{(1.4.10) Answer to Question 2.}

Proof of Theorem 1.3.10. Let $\varphi: G \times X \rightarrow H$ be an $\mathcal{M}$-measurable strict cocycle on the suitable measured Borel $G$-space $(X, \mu)$; we must show that there exists a Borel-measurable strict cocycle, $\psi$, such that $\psi$ is equivalent to $\varphi$. By Theorem 1.1.4, we may assume that $X$ is a Polish $G$-space. By Theorem 1.4.9, $\varphi$ is nearly essentially continuous. By Theorem 1.3.9, there exists a Borel-measurable almost cocycle, $\psi^{\prime}$, such that $\psi^{\prime}$ is equivalent to $\varphi$. As $\psi^{\prime}$ is equivalent to $\varphi, \psi^{\prime}$ is also nearly essentially continuous. The $(b) \Longrightarrow(a)$ implication of the Main Theorem (Theorem 1.4.7) tells us that $\psi^{\prime}$ is equivalent to a Borel-measurable strict cocyclecall it $\psi$. Clearly $\psi$ is equivalent to $\varphi$.

Remark 1.4.11. Let $\mathcal{M}$ be a useful pointclass. By Theorems 1.3.9 and 1.3.10, an $\mathcal{M}$ measurable strict (respectively, almost) cocycle is equivalent to a Borel-measurable strict (respectively, almost) cocycle. Parts (a), (b) and (c) of the Main Theorem are all properties which respect equivalence. So the Main Theorem remains true when all three occurrences of "Borel-measurable" are replaced by "M-Measurable". For example, the $C$-measurable version of the Main Theorem is true, and assuming $\boldsymbol{\Delta}_{n}^{1}$

sets are universally measurable and have the property of Baire, the $\boldsymbol{\Delta}_{n}^{1}$ version of the Main Theorem is true. The proof of the Main Theorem which is given in this paper does not seem to work for arbitrary useful $\mathcal{M}$; we must go via Theorems 1.3.9 and 1.3.10. In the specific case where $\mathcal{M}$ is $\boldsymbol{\Delta}_{n}^{1}$ for odd $n$, the proof of the Main Theorem given in this paper does work, but seems to require determinacy axioms (see [11]); these axioms are much stronger than the assumption that sets are measurable and have the property of Baire.

\section{Proofs}

In $\S \S 2.2-2.7$, we prove theorems which were stated in Section 1. Each of these six sections can be read independently of the other five. $\S 2.1$ contains preliminary material that is used in all six of the later sections. It contains many references to the literature. In $\S \S 2.2-2.7$, there are no further references to the literature; we have put whatever is needed into $\S 2.1$. 
We continue to use the notation and terminology of Section 1.

2.1. Preliminaries. We assume familiarity with the following: Polish spaces, the theory of Borel and analytic sets in these spaces, Baire-category, and elementary measure theory. All the prerequisites can be found in Kechris [7, which is also the reference for any unexplained notation and terminology. We also assume familiarity with Polish groups and their actions, in connection with Borel and analytic sets. The reference for unexplained notation and terminology is Becker and Kechris [3].

A. Topology. We frequently state - without proof - that some particular pointset is Borel or analytic. This can always be proved by the "quantifier-counting" methods of [7, Appendix C].

We treat Baire-category notions as quantifiers; that is, we use terminology such as "for comeager many $x \in A \ldots$ ", and use the following lemma for computing the complexity of pointsets.

Lemma 2.1.1 (see [7, 16.1 and 22.22]). (a) The class of Borel sets is closed under quantification of the form "for comeager many $y \in Y$ ", where $Y$ is a Polish space.

(b) The class of $G_{\delta}$ sets is closed under quantification of the form "for comeager many $y \in Y$ ", where $Y$ is a Polish space.

We often work with product spaces $W \times X$. Given a set $A \subset W \times X$, we denote the sections of $A$ as follows:

$$
\begin{aligned}
& A_{w}=\{x \in X:(w, x) \in A\}, \\
& A^{x}=\{w \in W:(w, x) \in A\} .
\end{aligned}
$$

Similarly, given a function $F: W \times X \rightarrow Y, F_{w}: X \rightarrow Y$ is the function $F_{w}(x)=$ $F(w, x)$ and $F^{x}: W \rightarrow Y$ is the function $F^{x}(w)=F(w, x)$.

Lemma 2.1.2 (see [7, 18.7]). Let $X$ and $Y$ be Polish spaces, let $R \subset X \times Y$ be Borel, and suppose that for all $x \in X, R_{x}$ is comeager. Then there exists a Borelmeasurable function $f: X \rightarrow Y$ which uniformizes $R$, i.e., for all $x \in X,(x, f(x)) \in$ $R$.

Lemma 2.1.3 (see [7, 3.8]). Let $X$ and $Y$ be Polish spaces, let $d$ be a metric on $Y$, let $A$ be an arbitrary dense subset of $X$, and let $F: A \rightarrow Y$ be continuous. For all $\varepsilon>0$, define

$$
\begin{aligned}
S_{\epsilon}= & \{x \in X: \text { there exists an open neighborhood } N \text { of } X, \text { with } x \in N, \\
& \text { such that for all points } \left.x_{1} \text { and } x_{2} \text { in } N \cap A, d\left(F\left(x_{1}\right), F\left(x_{2}\right)\right)<\varepsilon\right\},
\end{aligned}
$$

and define $S=\bigcap\left\{S_{\varepsilon}: \varepsilon>0\right\}$. Then $S$ is a $G_{\delta}, A \subset S$ and there exists a continuous function $\widetilde{F}: S \rightarrow Y$ such that $\widetilde{F}\lceil A=F$.

B. Measure. We also treat notions of measure as quantifiers.

Lemma 2.1.4 (see [7, 17.25 and 22.25]). Let $X$ be a standard Borel space, and let $\mu$ be a Borel probability measure on $X$. The class of Borel sets is closed under quantification of the form "for $\mu$-a.e. $x \in X$ ". 
Let $\lambda$ denote Lebesgue measure on $[0,1]$. For any Borel $A \subset[0,1]$ and any point $x \in[0,1]$, we say that $A$ has density 1 at $x$ when for all $\varepsilon>0$, there exists an $n \in \mathbb{N}$ such that for all $\delta$ in $(0,1 / n)$,

$$
\frac{\lambda(A \cap[x-\delta, x+\delta])}{2 \delta}>1-\varepsilon .
$$

Theorem 2.1.5, below, is a form of the Lebesgue Density Theorem. A proof can be found in [12, 3.20].

Theorem 2.1.5. For any Borel set $A \subset[0,1]$, for $\lambda$-a.e. $x \in A, A$ has density 1 at $x$.

The next two lemmas have the unusual property that they involve a relationship between measure and category.

Lemma 2.1.6. Let $W, X$ and $Y$ be Polish spaces, let $\mu$ be a Borel probability measure on $X$, let $F: W \times X \rightarrow Y$ be a Borel-measurable function, and let $\varepsilon>0$. There exists a compact set $K \subset X$ such that $\mu(K)>1-\varepsilon$ and there exists a Borel set $B \subset W \times K$ satisfying the following two properties.

(a) For all $x \in K$, for comeager many $w \in W,(w, x) \in B$.

(b) $F\lceil B$ is continuous.

Proof. Let $\left\{M_{i}\right\}$ and $\left\{N_{j}\right\}$ be countable bases for $W$ and $Y$, respectively. Let $\varepsilon_{j}$ be a sequence of positive numbers such that $\sum \varepsilon_{j}<\varepsilon$.

Claim. For all $j \in \mathbb{N}$, there exists a compact $K_{j} \subset X$ such that $\mu\left(K_{j}\right)>1-\varepsilon_{j}$, and there exists a Borel $B_{j} \subset W \times K_{j}$ satisfying the following two properties.

(a) For all $x \in K_{j}$, for comeager many $w \in W,(w, x) \in B_{j}$.

(b) $F^{-1}\left[N_{j}\right] \cap B_{j}$ is relatively open in $B_{j}$.

Assuming the Claim, we set $K=\bigcap_{j} K_{j}$ and $B=\bigcap_{j} B_{j}$. This lemma then follows easily from the Claim.

To prove the Claim, fix $j$. Define $D \subset X \times \mathbb{N}$ and $E \subset W \times X$ as follows:

$$
\begin{gathered}
D=\left\{(x, i): \text { for comeager many } w \in W, \text { if } w \in M_{i}, \text { then } F(w, x) \in N_{j}\right\} ; \\
E=\left\{(w, x):(\exists i \in \mathbb{N})\left[(x, i) \in D \text { and } w \in M_{i} \text { and } F(w, x) \in N_{j}\right]\right. \text { or }
\end{gathered}
$$$$
\left.\left[\left((\forall i \in \mathbb{N})\left(\text { if }(x, i) \in D \text {, then } w \notin M_{i}\right)\right) \text { and } F(w, x) \notin N_{j}\right]\right\} \text {. }
$$

By Lemma 2.1.1(a), $D$ is Borel, hence $E$ is also Borel. Note that for all $x \in X, E^{x}$ is comeager. So after we find $K_{j}$, if we set

$$
B_{j}=E \cap\left(W \times K_{j}\right),
$$

then part (a) of the Claim will hold. Note also that for all $x \in X$,

$$
\left(F^{x}\right)^{-1}\left[N_{j}\right] \cap E^{x}=\left(\bigcup\left\{M_{i}: i \in D_{x}\right\}\right) \cap E^{x} .
$$

Define $f: X \rightarrow 2^{\mathbb{N}}$ as follows: $f(x)$ is the characteristic function of $D_{x}$. As $D$ is Borel, $f$ is Borel-measurable, hence $\mu$-measurable. So there exists a compact $K_{j} \subset X$ such that $\mu\left(K_{j}\right)>1-\varepsilon_{j}$ and $f\left\lceil K_{j}\right.$ is continuous. This continuity, plus (2.1.7), implies that part (b) of the Claim holds.

Definition 2.1.8. Let $Y$ be a Polish space, let $d$ be a metric on $Y$, let $X$ be a standard Borel space, let $\mu$ be a Borel probability measure on $X$, and let $f$ and $f_{0}, f_{1}, f_{2}, \ldots$ be Borel-measurable functions from $X$ into $Y$. The sequence $f_{n}$ 
converges to $f$ in measure (with respect to $\mu$ and $d$ ) when for all $\varepsilon>0$, the following sequence of real numbers converges to 0 :

$$
\mu\left(\left\{x \in X: d\left(f_{n}(x), f(x)\right) \geq \varepsilon\right\}\right) .
$$

Lemma 2.1.9. Let $W$ and $Y$ be Polish spaces, let $d$ be a metric on $Y$ with finite diameter, let $X$ be a standard Borel space, let $\mu$ be a Borel probability measure on $X$, and let $F: W \times X \rightarrow Y$ be a Borel-measurable function. There exists a comeager subset $C$ of $W$ with the property that for every sequence of points $w_{n}$ from $C$ and any point $w \in C$,

$$
\begin{aligned}
& \text { if } w_{n} \text { converges to } w \text {, then } F_{w_{n}} \text { converges to } \\
& F_{w} \text { in measure (with respect to } \mu \text { and } d \text { ). }
\end{aligned}
$$

Proof. Without loss of generality, $d$ is a complete metric. For any Borel-measurable function $f: X \rightarrow Y$, let $[f]$ denote the equivalence class of $f$ with respect to equality $\mu$-almost everywhere. Let

$$
Z=\{[f]: f \text { is a Borel-measurable function from } X \text { into } Y\} .
$$

Define a metric, $\delta$, on $Z$ by

$$
\delta([f],[g])=\int d(f(x), g(x)) d \mu .
$$

It is well known that $\langle Z, \delta\rangle$ is a Polish space and that $f_{n}$ converges to $f$ in measure iff $\left[f_{n}\right]$ converges to $[f]$ in $\langle Z, \delta\rangle$.

Let $\widehat{F}: W \rightarrow Z$ be the function $\widehat{F}(w)=\left[F_{w}\right]$. It follows from the definition of $\delta$ and the Borel-measurability theorem in [7, 17.25], that $\widehat{F}$ is Borel-measurable. So $\widehat{F}$ is Baire-measurable; therefore (see [7, 8.38]), there exists a comeager set $C \subset W$ such that $\widehat{F}\lceil C$ is continuous.

C. Groups. The group operation is written multiplicatively, and $e$ denotes the group identity (of all groups). The groups under consideration are, with the exception noted below, always Polish topological groups. A metric on the group means a metric compatible with that topology. The metric is not necessarily complete.

The one exception is that we sometimes consider countable subgroups of a Polish group (topologized as a subspace).

Definition 2.1.10. Let $G$ be a Polish group, and let $d$ be a metric on $G$. We call $d$ left-invariant (respectively, right-invariant) if for any $g_{1}, g_{2}$ and $h$ in $G$, $d\left(h g_{1}, h g_{2}\right)=d\left(g_{1}, g_{2}\right)$ (respectively, $\left.d\left(g_{1} h, g_{2} h\right)=d\left(g_{1}, g_{2}\right)\right)$.

It is a theorem of Birkhoff and Kakutani (see [10, 1.22]) that any metrizable group admits a left-invariant metric. (Of course, it also admits a right-invariant metric; but, in general, there is no metric which is both left- and right-invariant.) The usual trick turns that metric into a metric of finite diameter, without affecting left- or right-invariance. Therefore, we have the following result.

Theorem 2.1.11. Let $G$ be a Polish group. There exists a left-invariant (or rightinvariant) metric $d$ on $G$ such that $d$-diam $(G) \leq 1$. 
Theorem 2.1.12. Let $G$ be a locally compact group. There exists a $\sigma$-finite Borel measure $\nu$ on $G$ satisfying both of the following properties.

(a) If $A \subset G$ is nonempty and open, then $\nu(A)>0$.

(b) If $B \subset G$ is Borel and $g \in G$, then $\nu(B)=\nu(B g)$.

A proof of Theorem 2.1.12 can be found in [4, page 142]. The measure $\nu$ is called right Haar measure.

Lemma 2.1.13 (see [7, 9.10]). Let $G$ and $H$ be Polish groups, and let $\pi: G \rightarrow H$ be a Borel-measurable homomorphism. Then $\pi$ is continuous.

D. $G$-spaces. Except in $\S 2.7$ (which is about the two concrete counterexamples), we have a Polish group, $G$, and a $G$-space, $X$, which are fixed throughout; and no other actions are under consideration. The $G$-space $X$ is sometimes a standard Borel $G$-space and sometimes a Polish $G$-space. Furthermore, we consider many different compatible topologies for $X$. We also consider various subsets of $X$, which are always viewed as topological spaces with the relative topology (for some given topology on $X$ ). These subspaces need not be Polish.

Definition 2.1.14. Let $G$ be a Polish group, and let $X$ be a standard Borel $G$ space. Given any set $P \subset X$, we define the set $Q(P) \subset G \times X$ as

$$
Q(P)=\{(g, x): x \in P \text { and } g \cdot x \in P\} .
$$

We next define the Vaught transforms, $A^{\triangle u}$ and $A^{* u}$. For more information on this topic see [3, 5.1.7].

Definition 2.1.15. Let $G$ be a Polish group, let $X$ be a standard Borel $G$-space, let $A \subset X$, and let $u$ be an open neighborhood of $G$. Define

$$
\begin{aligned}
A^{\triangle u} & =\{x \in X: \text { For a nonmeager-in- } u \text { set of } g, g \cdot x \in A\}, \\
A^{* u} & =\{x \in X: \text { For a comeager-in- } u \text { set of } g, g \cdot x \in A\} .
\end{aligned}
$$

By Lemma 2.1.1(a), if $A$ is Borel, so are $A^{\triangle u}$ and $A^{* u}$ for all open $u \subset G$. Clearly for any set $A, A^{\triangle G}$ is an invariant set.

Lemma 2.1.16. Let $G$ be a Polish group, let $X$ be a standard Borel $G$-space, and let $A$ and $B$ be disjoint invariant analytic subsets of $X$. There exists an invariant Borel set $C \subset X$ such that $A \subset C$ and $B \cap C=\emptyset$.

Proof. By the Lusin Separation Theorem (see [7, 14.7]), there exists a Borel set $D \subset X$ such that $A \subset D$ and $B \cap D=\emptyset$. Let $C=D^{\triangle G}$.

The rest of $\S 2.1 . D$ concerns compatible topologies (see Definition 1.1.3).

Theorem 2.1.17 (Becker and Kechris [3, 5.1.8]). Let $G$ be a Polish group, and let $X$ be a Polish $G$-space with topology $t$. For any Borel $A \subset X$ and any open $u \subset G$, there exists a compatible topology, $t^{\prime}$, for $X$ such that $t^{\prime}$ is finer than $t$ and such that $A^{\triangle u}$ is $t^{\prime}$-open. In particular, for any invariant Borel $A \subset X$, there exists a compatible topology, $t^{\prime}$, for $X$ such that $t^{\prime}$ is finer than $t$ and such that $A$ is $t^{\prime}$-open.

For some reason, the authors Becker and Kechris 3 neglected to explicitly state the following result.

Corollary 2.1.18. Let $G$ be a Polish group, let $X$ be a standard Borel G-space, and let $t_{0}, t_{1}, t_{2}, \ldots$ be a sequence of compatible topologies for $X$. There exists a compatible topology, $t^{\prime}$, for $X$ such that $t^{\prime}$ is finer than each of the $t_{n}$ 's. 
Proof. Given a sequence $\tau_{0} \subset \tau_{1} \subset \tau_{2} \subset \cdots$ of increasingly finer compatible topologies, $\bigcup_{n} \tau_{n}$ is also a Polish topology (see [7, 13.3]) and, therefore, a compatible topology. Suppose we are given a countable basis for each $t_{n}$; we need only-one at a time - make each of these countably many sets open in a compatible refinement of the existing compatible topology, and then take the union. So by Theorem 2.1.17, in order to prove Corollary 2.1.18, it will suffice to show that each $t_{n}$ has a countable basis consisting of sets of the form $A^{\triangle u}, A \subset X$ Borel, $u \subset G$ open. Let $B \subset X$ be $t_{n}$-open with $x \in B$. As the action is continuous with respect to $t_{n}$, there exists a $t_{n}$-open $A$ with $x \in A \subset B$, and there exists a symmetric open neighborhood $u$ of the group identity such that $u \cdot x \subset A$ and $u \cdot A \subset B$. Then $x \in A^{\triangle u} \subset B$. It is easy to see that $A^{\triangle u}$ is $t_{n}$-open.

Remark 2.1.19. When $X$ is a transitive Polish $G$-space, then by a theorem of Effros (see [4, page 251]), for fixed $x \in X$, the function $g \mapsto g \cdot x$ is an open mapping from $G$ to $X$. One consequence of this is that the compatible topology is unique. Another consequence is that sets of the form $A^{\triangle u}$ are open, i.e., the transitive case of Theorem 2.1.17. Because the topology is unique, the proofs of Theorem 1.2.12 and Theorem 1.4.6 (also known as Theorem C), given in $\S 2.3$ and $\S 2.6$, respectively, can be simplified in the transitive case. We do, however, prove the general case and leave the simplification to the reader. The reader who cares only about transitive actions should ignore all the changes in the topology.

E. Measured $G$-spaces. Let $(X, \mu)$ be a measured Borel $G$-space under the action $a: G \times X \rightarrow X$. Let MALG denote the measure algebra of $(X, \mu)$, which is a Polish space (complete metric: $\delta([P],[Q])=\mu(P \triangle Q)$ ). Since $\mu$ is quasi-invariant, the action $a$ induces an action $\hat{a}: G \times$ MALG $\rightarrow$ MALG. Consider the Polish group Aut* of Boolean automorphisms of MALG. The obvious action of Aut* on MALG is continuous. For details on all of the above, see [7, 17.2, 17.43, and 17.46]. As $\hat{a}$ acts by Boolean automorphisms, there exists a homomorphism $\pi: G \rightarrow$ Aut* associated with $\hat{a} . \pi$ is Borel-measurable, hence by Lemma 2.1.13, $\pi$ is continuous. Therefore, $\hat{a}$ is a continuous action. The next two lemmas follow easily from the continuity of $\hat{a}$.

Lemma 2.1.20. Let $G$ be a Polish group, and let $(X, \mu)$ be a measured Borel $G$-space. Let $g \in G$, and let $A_{n}$ be a sequence of Borel subsets of $X$. If $\mu\left(A_{n}\right)$ converges to 0 , then $\mu\left(g \cdot A_{n}\right)$ also converges to 0 .

Lemma 2.1.21. Let $G$ be a Polish group, and let $(X, \mu)$ be a measured Borel $G$ space. Let $A$ and $B$ be Borel subsets of $X$, let $g \in G$, and let $g_{n}$ be a sequence of elements of $G$. Suppose that for all $n \in \mathbb{N}, g_{n} \cdot A \subset B$ and that $g_{n}$ converges to $g$. Then for $\mu$-a.e. $x \in A, g \cdot x \in B$.

F. Cocycles. As mentioned at the beginning of $\S 2.1 . D$, except in $\S 2.7$ we have a fixed Polish group $G$ and a fixed $G$-space $X$. We also have a fixed quasi-invariant measure, $\mu$, on $X$, and a fixed target group, $H$. Therefore, we adopt the following conventions from now through $\S 2.6$. "Almost every" always refers to $\mu$. The term "strict cocycle" always means a Borel-measurable strict cocycle on the $G$-space $X$ with values in $H$. And the term "almost cocycle" always means a Borel-measurable almost cocycle on the measured $G$-space $(X, \mu)$ with values in $H$. 
Proposition 2.1.22. $\quad$ (a) Let $\varphi$ be a strict cocycle.

(i) For all $x \in X, \varphi(e, x)=e$.

(ii) For all $g \in G$ and for all $x \in X, \varphi\left(g^{-1}, g \cdot x\right)=[\varphi(g, x)]^{-1}$.

(b) Let $\psi$ be an almost cocycle.

(i) For a.e. $x \in X, \psi(e, x)=e$.

(ii) For all $g \in G$, for a.e. $x \in X, \psi\left(g^{-1}, g \cdot x\right)=[\psi(g, x)]^{-1}$.

2.2. Proofs of theorems about suitability. In $\S 2.2$, we prove Theorems 1.2.8, 1.2.9, and 1.2.11.

Proof of Theorem 1.2.8. Let $(X, \mu)$ be a measured Borel $G$-space which is suitable. We must prove that $(X, \mu)$ is weakly suitable. To prove this, let $Q \subset X$ be an almost invariant Borel set; we show below that there exists an invariant Borel set $Q^{\prime} \subset X$ such that $\mu\left(Q \triangle Q^{\prime}\right)=0$.

Almost invariance implies that, taking $M=G, A=Q$ and $B=X \backslash Q$, case (1) in the definition of suitable (Definition 1.2.7) is violated. So case (2) holds: there exist Borel sets $A^{\prime} \subset Q$ and $B^{\prime} \subset X \backslash Q$ such that $\mu\left(Q \backslash A^{\prime}\right)=\mu\left((X \backslash Q) \backslash B^{\prime}\right)=0$ and there exists an open neighborhood $N$ of the group identity such that $\left(N \cdot A^{\prime}\right) \cap B^{\prime}=\emptyset$.

Let $\mathcal{B}$ be a countable basis for $G$. Consider a fixed $g \in G$. Let $Y=\left(\left(g^{-1} \cdot A^{\prime}\right) \cap\right.$ $Q) \cup B^{\prime}$. Using the quasi-invariance of $\mu$ and the almost invariance of $Q$, we see that $\mu(Y)=1$. Let $M \in \mathcal{B}$ be such that $g \in M \subset N g$. Note that $Y \cap Q \subset g^{-1} \cdot A^{\prime}$, hence

$$
M \cdot(Y \cap Q) \subset M \cdot\left(g^{-1} \cdot A^{\prime}\right) \subset(N g) \cdot\left(g^{-1} \cdot A^{\prime}\right)=N \cdot A^{\prime} .
$$

Also note that $Y \backslash Q=B^{\prime}$. Therefore,

$$
[M \cdot(Y \cap Q)] \cap(Y \backslash Q)=\emptyset .
$$

Thus there is a $\mathcal{C} \subset \mathcal{B}$ such that $\bigcup\{M: M \in \mathcal{C}\}$ covers $G$, and for all $M \in \mathcal{C}$, there exists a Borel $Y^{M} \subset X$, with $\mu\left(Y^{M}\right)=1$, such that

$$
\left[M \cdot\left(Y^{M} \cap Q\right)\right] \cap\left(Y^{M} \backslash Q\right)=\emptyset .
$$

Let $\widehat{Y}=\bigcap\left\{Y^{M}: M \in \mathcal{C}\right\}$. Then $\mu(\widehat{Y})=1$ and the two invariant analytic sets $G \cdot(\widehat{Y} \cap Q)$ and $G \cdot(\widehat{Y} \backslash Q)$ are disjoint. By Lemma 2.1.16, there exists an invariant Borel set $Q^{\prime}$ which includes $G \cdot(\widehat{Y} \cap Q)$ and is disjoint from $G \cdot(\widehat{Y} \backslash Q)$. Clearly $\mu\left(Q \triangle Q^{\prime}\right)=0$.

Proof of Theorem 1.2.9. Let $G$ be a locally compact group, and let $(X, \mu)$ be a measured Borel $G$-space. We must prove that $(X, \mu)$ is suitable. To prove this, fix Borel sets $A, B \subset X$ which violate case (1) in the definition of suitable (Definition 1.2.7), and let $M$ be a counterexample to case (1); we show below that case (2) of that definition is satisfied.

Let $N$ be a symmetric open neighborhood of $e$ such that $N N \subset M$. Let $D$ be a countable dense subset of $N$. Let $C=D \cdot A$.

Claim. (a) For all $g \in N$, for $\mu$-a.e. $x \in A, g \cdot x \in C$.

(b) For all $g \in N$, for $\mu$-a.e. $x \in B, g \cdot x \notin C$.

Proof of Claim. By definition of $C$, (a) holds for all $g \in D$; as $D$ is dense in $N$, Lemma 2.1.21 implies that (a) holds for all $g \in N$. To prove (b), suppose it is false: fix $g \in N$ and a Borel $\widehat{B} \subset B$ such that $\mu(\widehat{B})>0$ and $g \cdot \widehat{B} \subset C$. Let $\widehat{C}=g \cdot \widehat{B}$. By quasi-invariance, $\mu(\widehat{C})>0$. Since $D$ is countable, there exists a Borel $\widetilde{C} \subset \widehat{C}$ such that $\mu(\widetilde{C})>0$ and there exists a $\tilde{g} \in D^{-1} \subset N^{-1}$ such that $\tilde{g} \cdot \widetilde{C} \subset A$. Let 
$\widetilde{A}=\tilde{g} \cdot \widetilde{C}$. Again using quasi-invariance, $\mu(\widetilde{A})>0$. Now $g^{-1} \tilde{g}^{-1} \in N^{-1} N \subset M$ and

$$
\left(g^{-1} \tilde{g}^{-1}\right) \cdot \widetilde{A}=g^{-1} \cdot\left(\tilde{g}^{-1} \cdot \widetilde{A}\right)=g^{-1} \cdot \widetilde{C} \subset g^{-1} \cdot \widehat{C}=\widehat{B} \subset B .
$$

That is, $g^{-1} \tilde{g}^{-1}$ witnesses that $M$ satisfies case (1) in the definition of suitable, a contradiction which proves the Claim.

Let $\nu$ denote right Haar measure on $G$, that is, a measure satisfying Theorem 2.1.12. Let

$$
\begin{aligned}
& A^{\prime}=\{x \in A: \text { for } \nu \text {-a.e. } g \in N, g \cdot x \in C\}, \\
& B^{\prime}=\{x \in B: \text { for } \nu \text {-a.e. } g \in N, g \cdot x \notin C\} .
\end{aligned}
$$

By Lemma 2.1.4, $A^{\prime}$ and $B^{\prime}$ are Borel. By the Claim and Fubini's Theorem,

$$
\mu\left(A \backslash A^{\prime}\right)=\mu\left(B \backslash B^{\prime}\right)=0 .
$$

To complete the proof, assume, towards a contradiction, that $\left(N \cdot A^{\prime}\right) \cap B^{\prime} \neq \emptyset$. Fix $h \in N$ and $x \in A^{\prime}$ such that $h \cdot x \in B^{\prime}$. By definition of $B^{\prime}$ and the invariance of Haar measure, for $\nu$-a.e. $g \in N h, g \cdot x \notin C$. By definition of $A^{\prime}, \nu(N h \cap N)=0$. As $N h \cap N$ is open, $N h \cap N=\emptyset$. But $e \in N$ and $h \in N$.

Proof of Theorem 1.2.11. Let $(X, \mu)$ be a weakly suitable measured Borel $G$-space, and let $A \subset X$ be a Borel set. We must prove that there exists a Borel set $B \subset A$ with $\mu(B)=\mu(A)$ and there exists a fulfillment of $B$.

Let $D$ be a countable dense subgroup of $G$, and let $\bar{g}=g_{0}, g_{1}, g_{2}, \ldots$ be an enumeration of $D$. Let $Q=D \cdot A$. Since $D$ is countable, $Q$ is a Borel set. Since $D$ is a group, for all $g_{n} \in D, g_{n} \cdot Q=Q$; and since $D$ is dense, Lemma 2.1.21 tells us that for all $g \in G$, for a.e. $x \in Q, g \cdot x \in Q$. That is, $Q$ is almost invariant. By definition of weakly suitable (Definition 1.2.4), there exists an invariant Borel set $Q^{\prime} \subset X$ such that $\mu\left(Q \triangle Q^{\prime}\right)=0$. Let $B=A \cap Q^{\prime}$. Clearly $B$ is a Borel subset of $A$ and $\mu(B)=\mu(A)$.

For $i \in \mathbb{N}$, recursively define

$$
E_{i}=\left(g_{i}^{-1} \cdot B\right) \backslash\left(\bigcup_{j<i} E_{j}\right) .
$$

Then set $\bar{E}$ to be the sequence $E_{0}, E_{1}, E_{2}, \ldots$ and set $\widetilde{E}=\bigcup_{i} E_{i}$. It is obvious that each $E_{i}$ is Borel and $(\bar{E}, \bar{g})$ satisfy the first two conditions in the definition of fulfillment (Definition 1.2.10(a)). All that remains to be proved is the third condition of that definition: $\mu((G \cdot B) \backslash \widetilde{E})=0$.

Since $Q^{\prime}$ is invariant, $G \cdot B \subset Q^{\prime}$. Hence

$$
\widetilde{E}=\bigcup_{i}\left(g_{i}^{-1} \cdot B\right)=D \cdot B \subset G \cdot B \subset Q^{\prime} .
$$

It will therefore suffice to show that $\mu(\widetilde{E})=\mu\left(Q^{\prime}\right)$. Since $B \subset A, \mu(B)=\mu(A)$ and $D$ is countable, by quasi-invariance, $\mu(D \cdot B)=\mu(D \cdot A)$. Thus

$$
\mu(\widetilde{E})=\mu(D \cdot B)=\mu(D \cdot A)=\mu(Q)=\mu\left(Q^{\prime}\right) .
$$

2.3. Proof of the continuous fulfillment theorem. In $\S 2.3$, we prove Theorem 1.2.12. Throughout this section, we have fixed a Polish group, $G$, and a suitable measured Borel $G$-space, $(X, \mu)$. We consider many different compatible topologies for $X$. (For the transitive case, see Remark 2.1.19.) 
Lemma 2.3.1. (a) Let $A_{0}, A_{1}, \ldots, A_{n}$ be subsets of $X$, let $t_{0}, t_{1}, \ldots, t_{n}$ be compatible topologies for $X$, and let $t^{*}$ be a compatible topology which is finer than each $t_{i}$ for $i \leq n$. Suppose that for all $i \leq n, \mu\left(t_{i}\right.$-bdry $\left.\left(A_{i}\right)\right)=0$. Then $\mu\left(t^{*}\right.$-bdry $\left.\left(A_{0} \cap A_{1} \cap \cdots \cap A_{n}\right)\right)=0$.

(b) Let $t$ be a compatible topology for $X$, and let $A_{1}$ and $A_{2}$ be $t$-closed subsets of $X$ such that $\mu\left(A_{1} \cap A_{2}\right)=0$. There exists a compatible topology $t^{*}$ such that $t^{*}$ is finer than $t$, and there exist two $t^{*}$-open sets, $V_{1}$ and $V_{2}$, such that

(i) $V_{1} \cap V_{2}=\emptyset$,

(ii) $\mu\left(A_{1} \backslash V_{1}\right)=\mu\left(A_{2} \backslash V_{2}\right)=0$,

(iii) $\mu\left(t^{*}\right.$-bdry $\left.\left(V_{1}\right)\right)=\mu\left(t^{*}\right.$-bdry $\left.\left(V_{2}\right)\right)=0$.

Proof. (a) The $t^{*}$-boundary of $A_{i}$ is a subset of the $t_{i}$-boundary of $A_{i}$. And the $t^{*}$-boundary of the intersection is a subset of the union of the $t^{*}$-boundaries of the $A_{i}$ 's.

(b) As $\langle X, t\rangle$ is a Polish space, there exists a set $Z \subset X$ such that $\mu(Z)=1$ and $\langle Z, t\rangle$ has a countable clopen basis. Let $Y=Z \backslash\left(A_{1} \cap A_{2}\right)$. Then $\mu(Y)=1$. Since $\langle Y, t\rangle$ also has a countable clopen basis, the two disjoint closed sets $A_{1} \cap Y$ and $A_{2} \cap Y$ can be separated by a clopen set $C \subset Y: A_{1} \cap Y \subset C$ and $A_{2} \cap Y \subset(Y \backslash C)$. Since $C$ is clopen in the relative topology, there exist two $t$-open subsets of $X, W_{1}$ and $W_{2}$, such that $C \subset W_{1} \subset[C \cup(X \backslash Y)]$ and $(Y \backslash C) \subset W_{2} \subset[(Y \backslash C) \cup(X \backslash Y)]$. First note that

$$
\mu\left(W_{1} \cap W_{2}\right)=\mu\left(A_{1} \backslash W_{1}\right)=\mu\left(A_{2} \backslash W_{2}\right)=0,
$$

because all three of the above sets are subsets of $X \backslash Y$. Second, note that $Y \subset$ $W_{1} \cup W_{2}$.

Let $D \subset X$ be the maximal $t$-open measure 0 subset of $X$. $D$ is invariant, so by Theorem 2.1.17, there exists a compatible topology $t^{*}$ for $X$ such that $t^{*}$ is finer than $t$ and such that $X \backslash D$ is $t^{*}$-open. Let $V_{1}=W_{1} \backslash D$ and $V_{2}=W_{2} \backslash D$. Clearly $V_{1}$ and $V_{2}$ are $t^{*}$-open. Since $W_{1} \cap W_{2}$ is a $t$-open set of measure $0, W_{1} \cap W_{2} \subset D$; hence (i) holds. Since $\mu\left(W_{i} \backslash V_{i}\right)=0$ and $\mu\left(A_{i} \backslash W_{i}\right)=0$, (ii) holds. Since $Y \subset W_{1} \cup W_{2}$, clearly $Y \backslash D \subset V_{1} \cup V_{2}$, and, therefore, $\mu\left(V_{1} \cup V_{2}\right)=1$. And $V_{1}$ and $V_{2}$ are disjoint $t^{*}$-open sets, so (iii) holds.

Lemma 2.3.2. Let $A$ be a Borel subset of $X$, let $M \subset G$ be an open neighborhood of $e$, and let $C$ be a countable dense subset of $M$. There exists a compatible topology, $t^{\prime}$, for $X$ and there exists a $t^{\prime}$-open set $V^{\prime} \subset X$ such that

(a) $\mu\left(A \backslash V^{\prime}\right)=0$,

(b) $\mu\left(V^{\prime} \backslash(C \cdot A)\right)=0$.

Proof. Let $B=X \backslash(C \cdot A)$. Then $B$ is Borel. Since $C$ is dense in $M$, by Lemma 2.1.21, for all $g \in M$, for a.e. $x \in A, g \cdot x \notin B$. That is, case (1) in the definition of suitable (Definition 1.2.7) is violated. So case (2) of that definition holds: there exist Borel sets $A^{\prime} \subset A$ and $B^{\prime} \subset B$ with $\mu\left(A \backslash A^{\prime}\right)=\mu\left(B \backslash B^{\prime}\right)=0$ and there exists an open neighborhood $N$ of $e$ such that $\left(N \cdot A^{\prime}\right) \cap B^{\prime}=\emptyset$.

Let $u$ be a symmetric open neighborhood of $e$ such that $u u \subset N$. Then $u \cdot A^{\prime} \cap$ $u \cdot B^{\prime}=\emptyset$. Both $u \cdot A^{\prime}$ and $u \cdot B^{\prime}$ are analytic sets, so there is a Borel set $D$ such that $u \cdot A^{\prime} \subset D$ and $\left(u \cdot B^{\prime}\right) \cap D=\emptyset$. Let $V^{\prime}=D^{\triangle u}$ (see Definition 2.1.15). By Theorems 1.1.4 and 2.1.17, there exists a compatible topology, $t^{\prime}$, for $X$ such that $V^{\prime}$ is $t^{\prime}$-open. 
Using the definition of the Vaught $\triangle$-transform and the fact that $u \cdot A^{\prime} \subset D$, we see that

$$
A^{\prime} \subset\left(u \cdot A^{\prime}\right)^{\triangle u} \subset D^{\triangle u}=V^{\prime} .
$$

And $\mu\left(A \backslash A^{\prime}\right)=0$, which proves (a).

Using the definitions of both Vaught transforms, and the fact that $\left(u \cdot B^{\prime}\right) \subset$ $(X \backslash D)$, we see that

$$
B^{\prime} \subset\left(u \cdot B^{\prime}\right)^{* u} \subset(X \backslash D)^{* u}=X \backslash\left(D^{\triangle u}\right)=X \backslash V^{\prime} .
$$

Hence $B^{\prime} \cap V^{\prime}=\phi$, and, therefore,

$$
V^{\prime} \backslash(C \cdot A)=V^{\prime} \cap(X \backslash(C \cdot A))=V^{\prime} \cap B=V^{\prime} \cap\left(B \backslash B^{\prime}\right) \subset B \backslash B^{\prime} .
$$

And $\mu\left(B \backslash B^{\prime}\right)=0$, which proves (b).

Lemma 2.3.3. Let $t$ be a compatible topology for $X$, let $A$ be a $t$-closed subset of $X$, let $M \subset G$ be an open neighborhood of e, and let $C$ be a countable dense subset of $M$.

(a) There exists a compatible topology, $t^{*}$, for $X$ such that $t^{*}$ is finer than $t$, and there exists a $t^{*}$-open set $V \subset X$ such that

(i) $\mu(A \backslash V)=0$,

(ii) $\mu(V \backslash(C \cdot A))=0$,

(iii) $\mu\left(t^{*}-b d r y(V)\right)=0$.

(b) Suppose that $C$ is also a subgroup of $G$. Then there exists a compatible topology, $\tilde{t}$, for $X$ such that $\tilde{t}$ is finer than $t$, and there exists a $\tilde{t}$-open set $V \subset X$ such that $\mu((C \cdot A) \triangle V)=0$.

Proof. By Lemma 2.3.2, there exists a compatible topology, $t^{\prime}$, and a $t^{\prime}$-open $V^{\prime} \subset$ $X$, such that $\mu\left(A \backslash V^{\prime}\right)=0$ and $\mu\left(V^{\prime} \backslash(C \cdot A)\right)=0$. By Corollary 2.1.18, there exists a compatible topology, $\tilde{t}$, which is finer than both $t$ and $t^{\prime}$.

(a) We now apply Lemma 2.3.1(b) to the two $\tilde{t}$-closed sets $A$ and $X \backslash V^{\prime}$ : there exists a compatible topology $t^{*}$ finer than $\tilde{t}$ and there exist two $t^{*}$-open sets, $V_{1}$ and $V_{2}$, such that $V_{1} \cap V_{2}=\emptyset, \mu\left(A \backslash V_{1}\right)=0, \mu\left(\left(X \backslash V^{\prime}\right) \backslash V_{2}\right)=0$, and $\mu\left(t^{*}\right.$-bdry $\left.\left(V_{1}\right)\right)=0$. Let $V=V_{1}$. Clearly (i) and (iii) hold. Since $V \cap V_{2}=\emptyset$ and $\mu\left(\left(X \backslash V^{\prime}\right) \backslash V_{2}\right)=0, \mu\left(V \backslash V^{\prime}\right)=0$. And $\mu\left(V^{\prime} \backslash(C \cdot A)\right)=0$, which gives us (ii).

(b) Let $V=C \cdot V^{\prime}$. As $\tilde{t}$ is a compatible topology, the fact that $V^{\prime}$ is $\tilde{t}$-open implies that $V$ is also $\tilde{t}$-open. Quasi-invariance, together with the fact that $\mu\left(A \backslash V^{\prime}\right)=0$, implies that $\mu((C \cdot A) \backslash V)=0$. Since $C$ is a group, quasi-invariance, together with the fact that $\mu\left(V^{\prime} \backslash(C \cdot A)\right)=0$, implies that $\mu(V \backslash(C \cdot A))=0$.

The collection of finite sequences from $\mathbb{N}$ is denoted $\mathbb{N}<\mathbb{N}$; and $\mathbb{N}<m$ denotes the sequences of length less than $m$.

Definition 2.3.4. (a) Let $\sigma \in \mathbb{N}^{<\mathbb{N}}, \sigma$ is called regular if either $\sigma=\langle\rangle$ or the last coordinate of $\sigma$ is not 0 .

(b) Given $\sigma \in \mathbb{N}^{<\mathbb{N}}, \tilde{\sigma}$ denotes the longest regular initial segment of $\sigma$.

(c) For $n \in \mathbb{N}, \rho_{n}$ denotes the length $n$ sequence of 1's.

(d) For regular $\sigma, \tau \in \mathbb{N}<\mathbb{N}$, we call $\sigma$ and $\tau$ equivalent if there exists an $n \in \mathbb{N}$ such that either $\sigma=\tau^{\wedge} \rho_{n}$ or $\tau=\sigma^{\wedge} \rho_{n}$.

Lemma 2.3.5. Let $\hat{t}$ be a compatible topology for $X$, let $F$ be a $\hat{t}$-closed subset of $X$, let $M_{0}, M_{1}, M_{2}, \ldots$ be a sequence of symmetric open neighborhoods of the group identity of $G$, with $M_{0}=G$, and let $D$ be a countable dense subgroup of $G$. There 
exists a sequence $t_{0}, t_{1}, t_{2}, \ldots$ of compatible topologies for $X$, with $t_{0}$ finer than $\hat{t}$, there exists a nonempty subtree $T$ of $\mathbb{N}<\mathbb{N}$, for every $\sigma \in T$, there exists a Borel set $V_{\sigma} \subset X$, with $\mu\left((D \cdot F) \backslash V_{\langle\rangle}\right)=0$, and for every regular $\sigma \in T$, there exists a Borel set $U_{\sigma} \subset X$, with $U_{\langle\rangle}=F$, such that if $T_{n}$ denotes $T \cap \mathbb{N}<(n+1)$, then for all $n \in \mathbb{N}$, all 15 of the following properties hold.

(1) $t_{n+1}$ is finer than $t_{n}$.

(2) $T_{n}$ is finite.

(3) For all $\sigma \in T_{n}, \sigma^{\frown}(0) \in T_{n+1}$.

(4) For all $\sigma \in T_{n}, \sigma^{\frown}(1) \in T_{n+1}$ iff $\sigma$ is regular.

(5) For all regular $\sigma \in T_{n}, U_{\sigma}$ is $t_{n}$-closed.

(6) For all regular $\sigma, \tau \in T_{n}$, if $\sigma$ is equivalent to $\tau$, then $U_{\sigma}=U_{\tau}$.

(7) For all regular $\sigma, \tau \in T_{n}$, if $\sigma$ is not equivalent to $\tau$, then $U_{\sigma} \cap U_{\tau}=\emptyset$.

(8) $\mu\left(\bigcup\left\{U_{\sigma}: \sigma \in T_{n}, \sigma\right.\right.$ regular $\left.\}\right) \geq \mu(D \cdot F)-\frac{1}{n}$.

(9) For all $i>0$, for all $\sigma^{\frown}(i) \in T_{n+1}$, if $m=$ length $(\tilde{\sigma})$, then there exists an $h \in M_{m}$ such that $h \cdot U_{\sigma \frown(i)} \subset U_{\tilde{\sigma}}$.

(10) For all $\sigma \in T_{n}, V_{\sigma}$ is $t_{n}$-open.

(11) For all $\sigma^{\frown}(i) \in T_{n+1}, V_{\sigma \frown(i)} \subset V_{\sigma}$.

(12) For all $i, j \in \mathbb{N}$, if $i \neq j$ and $\sigma^{\frown}(i), \sigma^{\frown}(j) \in T_{n+1}$, then $V_{\sigma \frown(i)} \cap V_{\sigma \frown(j)}=\emptyset$.

(13) $\mu\left(\bigcup\left\{V_{\sigma}: \sigma \in T_{n}\right.\right.$, length $\left.\left.(\sigma)=n\right\}\right)=\mu(D \cdot F)$.

(14) For all regular $\sigma \in T_{n}, \mu\left(U_{\sigma} \backslash V_{\sigma}\right)=0$.

(15) For all regular $\sigma \in T_{n}$ such that length $(\sigma)=n$, there exists a countable subset $C$ of $M_{n}$ such that $\mu\left(V_{\sigma} \backslash\left(C \cdot U_{\sigma}\right)\right)=0$.

Proof. By induction on $n$, we simultaneously construct $t_{n}, T_{n}$, all the $V_{\sigma}$ 's for $\sigma \in T_{n}$ with length $(\sigma)=n$ and all the $U_{\sigma}$ 's for regular $\sigma \in T_{n}$ with length $(\sigma)=n$, and we prove that the newly constructed objects satisfy $(1)-(15)$.

Of course, we start at stage 0 . Set $T_{0}=\{\langle\rangle\}$ and $U_{\langle\rangle}=F$. Lemma 2.3.3(b) (taking $A=F, M=G$ and $C=D$ ) now gives us a compatible topology, $t_{0}$, which is finer than $\hat{t}$ and a $t_{0}$-open $V_{\langle\rangle}$such that $\mu\left((D \cdot F) \triangle V_{\langle\rangle}\right)=0$. It is easy to verify that all necessary properties are satisfied in the $n=0$ case.

Let us now assume the induction hypothesis: the construction has been completed out to stage $n$. The rest of the proof of this lemma consists of the stage $n+1$ construction.

Let $U^{*}=\bigcup\left\{U_{\sigma}: \sigma \in T_{n}, \sigma\right.$ regular $\}$. Call a set $K \subset X \operatorname{good}$ if it satisfies all four of the following conditions.

(a) $K$ is $t_{n}$-compact.

(b) $\mu(K)>0$.

(c) $K \cap U^{*}=\emptyset$

(d) There exists a $\sigma \in T_{n}$ such that length $(\sigma)=n$ and

(i) $K \subset V_{\sigma}$,

(ii) if $m=\operatorname{length}(\tilde{\sigma})$, then there exists a $g \in M_{m}$ such that $K \subset g \cdot U_{\tilde{\sigma}}$.

Let $\mathcal{F}$ be a maximal family of pairwise disjoint good sets.

Claim. $\mu\left((\bigcup \mathcal{F}) \cup U^{*}\right) \geq \mu(D \cdot F)$.

Proof of Claim. Suppose this Claim is false. By (13) of the induction hypothesis, there is a $\sigma \in T_{n}$ with length $(\sigma)=n$ such that

$$
\mu\left(V_{\sigma} \backslash\left((\bigcup \mathcal{F}) \cup U^{*}\right)\right)>0 .
$$


Now $m=\operatorname{length}(\tilde{\sigma}) \leq n$, so (15) of the induction hypothesis holds at $m$ : there exists a countable subset $C$ of $M_{m}$ such that $\mu\left(V_{\tilde{\sigma}} \backslash\left(C \cdot U_{\tilde{\sigma}}\right)\right)=0$. By (11) of the induction hypothesis, $V_{\sigma} \subset V_{\tilde{\sigma}}$, hence $\mu\left(V_{\sigma} \backslash\left(C \cdot U_{\tilde{\sigma}}\right)\right)=0$. Therefore,

$$
\mu\left(\left(\left(C \cdot U_{\tilde{\sigma}}\right) \cap V_{\sigma}\right) \backslash\left((\bigcup \mathcal{F}) \cup U^{*}\right)\right)>0 .
$$

As $C$ is countable, there exists a fixed $g \in M_{m}$ such that

$$
\mu\left(\left(\left(g \cdot U_{\tilde{\sigma}}\right) \cap V_{\sigma}\right) \backslash\left((\bigcup \mathcal{F}) \cup U^{*}\right)\right)>0 .
$$

Let $K$ be a $t_{n}$-compact, positive-measure subset of

$$
\left(\left(g \cdot U_{\tilde{\sigma}}\right) \cap V_{\sigma}\right) \backslash\left((\bigcup \mathcal{F}) \cup U^{*}\right) .
$$

Then $K$ is good and disjoint from $\bigcup \mathcal{F}$, contradicting the maximality of $\mathcal{F}$. This proves the Claim.

The Claim ensures that there is a finite subfamily $\mathcal{F}^{*}$ of $\mathcal{F}$ such that

$$
\mu\left(\left(\bigcup \mathcal{F}^{*}\right) \cup U^{*}\right) \geq \mu(D \cdot F)-\frac{1}{n+1} .
$$

For $\sigma \in T_{n}$ with length $(\sigma)=n$, let $\mathcal{F}_{\sigma}^{*}=\left\{K \in \mathcal{F}^{*}: K \subset V_{\sigma}\right\}$, and let $a_{\sigma}$ denote the number of elements of $\mathcal{F}_{\sigma}^{*}$ (which may be 0 ). By (d)(i) in the definition of good, all members of $\mathcal{F}^{*}$ are in at least one $\mathcal{F}_{\sigma}^{*}$; and by (11) and (12) of the induction hypothesis, all members of $\mathcal{F}^{*}$ are in at most one $\mathcal{F}_{\sigma}^{*}$.

The tree $T_{n+1}$ is obtained from $T_{n}$ as follows: For each regular $\sigma \in T_{n}$ of length $n, \sigma$ has $a_{\sigma}+2$ length 1 extensions in $T_{n+1}$, whose last coordinates go from 0 through $a_{\sigma}+1$. For each nonregular $\sigma \in T_{n}$ of length $n, \sigma$ has $a_{\sigma}+1$ length 1 extensions in $T_{n+1}$, whose last coordinates go from 0 through $a_{\sigma}+1$, excluding 1 . Then $T_{n+1}$ satisfies (2)-(4).

We now construct the $U$ 's at stage $n+1$. For regular $\sigma \in T_{n}$ of length $n$, set $U_{\sigma \frown(1)}=U_{\sigma}$. For any $\sigma \in T_{n}$ of length $n$, set $\left\{U_{\sigma \frown(i)}: 2 \leq i \leq a_{\sigma}+1\right\}$ to be the members of $\mathcal{F}_{\sigma}^{*}$. This completes the construction of the $U$ 's. Obviously, this construction gives us the stage $n+1$ case of (6). This construction, together with (c) in the definition of good, the definition of $\mathcal{F}$ and (7) of the induction hypothesis gives us the stage $n+1$ case of (7); and together with (2.3.6) gives us the stage $n+1$ case of (8). We next verify that (9) holds at stage $n+1$ for $\sigma \in T_{n}$ of length $n$. There are two cases. One case is $\sigma^{\frown}(1)$. In this case, $\sigma$ is regular, so $\sigma=\tilde{\sigma}$ and $U_{\sigma \sim(1)}=U_{\sigma}$. As $M_{m}\left(=M_{n}\right)$ is a neighborhood of $e$, we can take $h=e$. In the other case, by (d)(ii) in the definition of good, there exists a $g \in M_{m}$ such that $U_{\sigma \frown(i)} \subset g \cdot U_{\tilde{\sigma}}$. As $M_{m}$ is symmetric, we can take $h=g^{-1}$. Finally, note that by (a) in the definition of good and (5) of the induction hypothesis, for all regular $\sigma \in T_{n+1}, U_{\sigma}$ is $t_{n}$-closed. Therefore, the $n+1$ case of (5) will follow from (1).

By (2) and (7), which have already been proved,

$$
\mathcal{U}=\left\{U_{\tau}: \tau \in T_{n+1}, \text { length }(\tau)=n+1, \tau \text { regular }\right\}
$$

is a finite collection of pairwise disjoint sets. And, as shown above, the members of this collection are $t_{n}$-closed. By Lemma 2.3.1(b), for any two distinct members, $U_{1}$ and $U_{2}$, of $\mathcal{U}$, there exists a compatible topology $t^{*}$ and there exist disjoint $t^{*}$-open sets $Y_{1}$ and $Y_{2}$, whose $t^{*}$-boundaries have measure 0 , with the property that $\mu\left(U_{1} \backslash Y_{1}\right)=\mu\left(U_{2} \backslash Y_{2}\right)=0$. Taking finite intersections and applying Corollary 2.1.18 and Lemma 2.3.1(a), we obtain a family

$$
\left\{t_{\tau}: \tau \in T_{n+1} \text {, length }(\tau)=n+1, \tau \text { regular }\right\}
$$


of compatible topologies, each finer than $t_{n}$, and we obtain a pairwise disjoint collection

$$
\left\{Q_{\tau}: \tau \in T_{n+1}, \text { length }(\tau)=n+1, \tau \text { regular }\right\}
$$

of subsets of $X$ such that for all such $\tau, Q_{\tau}$ is $t_{\tau}$-open, the $t_{\tau}$-boundary of $Q_{\tau}$ has measure 0 and $\mu\left(U_{\tau} \backslash Q_{\tau}\right)=0$.

We next construct the $V_{\tau}$ 's at stage $n+1$, for $\tau$ regular. Fix a regular $\tau \in$ $T_{n+1} \backslash T_{n}$. Let $\sigma$ be the initial segment of $\tau$ of length $n$. Fix a countable dense subset $C$ of $M_{n+1}$. By Lemma 2.3.3(a), applied to $A=U_{\tau}$, there exists a compatible topology $t^{\tau}$ for $X$, and there exists a $t^{\tau}$-open $V^{\tau} \subset X$ such that $\mu\left(U_{\tau} \backslash V^{\tau}\right)=$ $0, \mu\left(V^{\tau} \backslash\left(C \cdot U_{\tau}\right)\right)=0$, and $\mu\left(t^{\tau}\right.$-bdry $\left.\left(V^{\tau}\right)\right)=0$. Let $V_{\tau}=Q_{\tau} \cap V^{\tau} \cap V_{\sigma}$. This completes the construction of the $V^{\prime}$ 's for regular sequences. Clearly this construction gives us the stage $n+1$ case of (15), and for regular sequences gives us the stage $n+1$ case of (11) and (12). We next verify that $\mu\left(U_{\tau} \backslash V_{\sigma}\right)=0$; this fact implies that this construction gives us (14) at stage $n+1$. There are two cases. One case is $\tau=\sigma^{\frown}(1)$. In this case, $U_{\tau}=U_{\sigma}$, so the fact that $\mu\left(U_{\tau} \backslash V_{\sigma}\right)=0$ follows from (14) of the induction hypothesis. In the other case, by (d)(i) in the definition of good, $U_{\tau} \subset V_{\sigma}$. Finally, assuming that $t_{n+1}$ is finer than $t_{n}$ and also finer than each of the $t_{\tau}$ 's and $t^{\tau}$ 's, we obtain two additional facts. First the stage $n+1$ case of (10) holds for regular sequences. Second, using Lemma 2.3.1(a), for regular $\tau \in T_{n+1}$ of length $n+1$, the $t_{n+1}$-boundary of $\left(Q_{\tau} \cap V^{\tau}\right)$ has measure 0 .

By Corollary 2.1.18, there exists a compatible topology, $t_{n+1}$, which is finer than $t_{n}$ and also finer than $t_{\tau}$ and $t^{\tau}$ for all regular $\tau \in T_{n+1}$ of length $n+1$. Thus (1) holds, and, as previously mentioned, (5) holds and (10) holds for regular sequences.

To complete the proof, we construct the $V_{\tau}$ 's at stage $n+1$ for nonregular $\tau$, and verify (10)-(13). Fix $\sigma \in T_{n}$ such that length $(\sigma)=n$. For $i>0$ with $\sigma^{\frown}(i) \in T_{n+1}$, let $W_{i}=Q_{\sigma \frown(i)} \cap V^{\sigma \frown(i)}$, and let $F_{i}$ be the $t_{n+1}$-closure of $W_{i}$. Let

$$
V_{\sigma \frown(0)}=V_{\sigma} \backslash\left(\bigcup\left\{F_{i}: i>0, \sigma^{\frown}(i) \in T_{n+1}\right\}\right) .
$$

For $\sigma^{\frown}(0),(11)$ is trivial, and (10) follows trivially from (10) of the induction hypothesis, (1) and (2). For $i>0, V_{\sigma \frown(i)}$ is, by definition, $W_{i} \cap V_{\sigma}$. Hence (12) is satisfied for this $\sigma$. Recall that for $i>0$, the $t_{n+1}$-boundary of $W_{i}$ has measure 0 ; that is, $\mu\left(W_{i}\right)=\mu\left(F_{i}\right)$. Hence

$$
\begin{aligned}
\mu\left(V_{\sigma \frown(0)}\right) & =\mu\left(V_{\sigma} \backslash\left(\bigcup\left\{W_{i}: i>0, \sigma^{\frown}(i) \in T_{n+1}\right\}\right)\right) \\
& =\mu\left(V_{\sigma} \backslash\left(\bigcup\left\{V_{\sigma \frown(i)}: i>0, \sigma^{\frown}(i) \in T_{n+1}\right\}\right)\right) .
\end{aligned}
$$

By (11) and (12),

$$
\mu\left(V_{\sigma}\right)=\mu\left(\bigcup\left\{V_{\sigma \frown(i)}: \sigma^{\frown}(i) \in T_{n+1}\right\}\right) .
$$

Therefore, since $\sigma$ is an arbitrary element of $T_{n}$ of length $n$, the stage $n+1$ case of (13) follows from (13) of the induction hypothesis.

Lemma 2.3.7. Let $D$ be a countable dense subgroup of $G$, let $\hat{t}$ be a compatible topology for $X$, and let $F$ be a $\hat{t}$-closed subset of $X$ with the property that $\mu(D \cdot F)=$ $\mu(G \cdot F)$. There exists a compatible topology, $t$, for $X$, with $t$ finer than $\hat{t}$, and there exists a fulfilment $(\bar{E}, \bar{g})$ of $F$ whose fulfilment function $f: \widetilde{E} \rightarrow G$ is continuous with respect to $t$. 
Proof. By Theorem 2.1.11, there exists a left-invariant metric, $d$, on $G$ such that $d$ $\operatorname{diam}(G) \leq 1$. Let $M_{0}, M_{1}, M_{2}, \ldots$ be a sequence of symmetric open neighborhoods of the group identity such that $M_{0}=G$ and such that for all $n \in \mathbb{N}, d$-diam $\left(M_{n}\right) \leq$ $\frac{1}{2^{n}}$.

We now apply Lemma 2.3.5 to the above, and obtain $\left\{t_{n}: n \in \mathbb{N}\right\}, T$, $\left\{T_{n}: n \in \mathbb{N}\right\},\left\{U_{\sigma}: \sigma \in T, \sigma\right.$ regular $\}$, and $\left\{V_{\sigma}: \sigma \in T\right\}$ satisfying that lemma. In what follows, all citations of numbers (1)-(15) refer to the numbered properties of Lemma 2.3.5.

By Corollary 2.1.18, there is a compatible topology, $t$, for $X$ such that $t$ is finer than all the $t_{n}$ 's and, hence, finer than $\hat{t}$.

For $\tau=\sigma^{\frown}(i) \in T$, with $i>0$, let $m(\tau)=\operatorname{length}(\tilde{\sigma})$ and choose an $h_{\tau} \in M_{m(\tau)}$ such that $h_{\tau} \cdot U_{\tau} \subset U_{\tilde{\sigma}}$. Such an $h_{\tau}$ must exist, by (9). In light of (4) and (6), if $i=1$ we can take $h_{\tau}$ to be $e$. Let $h_{\langle\rangle}=e$. We have thus fixed an $h_{\tau} \in G$ for every regular $\tau \in T$.

Let $\widehat{G}$ be the subgroup of $G$ generated by the $h_{\tau}$ 's. Let $Y$ be the smallest subset of $X$ such that: for all regular $\sigma \in T, U_{\sigma} \backslash V_{\sigma} \subset Y$; and $\widehat{G} \cdot Y=Y$. By (14) and quasi-invariance, $\mu(Y)=0$. For regular $\sigma \in T$, let $E^{\sigma}=U_{\sigma} \backslash Y$. Thus each $E^{\sigma}$ is a Borel set and

$$
E^{\sigma} \subset U_{\sigma} \cap V_{\sigma} .
$$

Let $\bar{E}=E_{0}, E_{1}, E_{2}, \ldots$ be an enumeration of the countable collection of Borel sets $\left\{E^{\sigma}: \sigma \in T, \sigma\right.$ regular $\}$. (If this collection is finite, set the rest of the $E_{i}$ 's to be $\emptyset$.) Let $\widetilde{E}=\bigcup\left\{E_{i}: i \in \mathbb{N}\right\}$. For regular $\sigma \in T$, let $j(\sigma) \in \mathbb{N}$ be such that $E^{\sigma}=E_{j(\sigma)}$. By (6) and (7): the preimage of a point under $j$ is an equivalence class, and the $E_{i}$ 's are pairwise disjoint. Thus $\bar{E}$ satisfies (i) in the definition of fulfillment (Definition 1.2.10(a)).

Using (8) and the fact that $\mu(Y)=0$, we see that for all $n \in \mathbb{N}$,

$$
\begin{aligned}
\mu(\widetilde{E}) & =\mu\left(\bigcup\left\{E_{i}: i \in \mathbb{N}\right\}\right) \\
& =\mu\left(\bigcup\left\{E^{\sigma}: \sigma \in T, \sigma \text { regular }\right\}\right) \\
& =\mu\left(\bigcup\left\{U_{\sigma} \backslash Y: \sigma \in T, \sigma \text { regular }\right\}\right) \\
& =\mu\left(\bigcup\left\{U_{\sigma}: \sigma \in T, \sigma \text { regular }\right\}\right) \\
& \geq \mu\left(\bigcup\left\{U_{\sigma}: \sigma \in T_{n}, \sigma \text { regular }\right\}\right) \\
& \geq \mu(D \cdot F)-1 / n .
\end{aligned}
$$

Since $n$ is arbitrary, $\mu(\widetilde{E}) \geq \mu(D \cdot F)=\mu(G \cdot F)$. So assuming $\widetilde{E} \subset G \cdot F, \widetilde{E}$ satisfies (iii) in the definition of fulfillment. Therefore, (iii) will follow from (ii) of that definition.

We now define, for each regular $\sigma \in T$, a $g^{\sigma} \in G$. This definition is by recursion on length $(\sigma): g^{\langle\rangle}=h_{\langle\rangle}$; if $\tau$ is a length 1 extension of $\sigma$, then

$$
g^{\tau}=g^{\tilde{\sigma}} h_{\tau} .
$$

Since $h_{\sigma \frown(1)}$ was chosen to be $e$, if $\tau$ and $\tau^{*}$ are equivalent then $g^{\tau}=g^{\tau *}$.

We next define, for each $i \in \mathbb{N}$, a $g_{i} \in G$. If $i$ is in the image of the function $j$, set $g_{i}=g^{\tau}$ for some (equivalently, for any) regular $\tau \in T$ such that $j(\tau)=i$. This is well defined, since the preimage of $i$ under the function $j$ is an equivalence class. 
If $i$ is not in the image of $j$ (that is, we had a finite collection so we let $E_{i}=\emptyset$ for large $i$ ), set $g_{i}=e$. Let $\bar{g}$ denote the sequence $g_{0}, g_{1}, g_{2}, \ldots$.

Suppose $\tau=\sigma^{\frown}(i) \in T$, where $i>0$. Since $h_{\tau} \cdot U_{\tau} \subset U_{\tilde{\sigma}}$, by definition of $Y$ and of the $E^{\sigma}$ s, $h_{\tau} \cdot E^{\tau} \subset E^{\tilde{\sigma}}$. So a trivial induction on length $(\tau)$ establishes that for all regular $\tau \in T, g^{\tau} \cdot E^{\tau} \subset E^{\langle\rangle}$. Hence for all $i \in \mathbb{N}, g_{i} \cdot E_{i} \subset E^{\langle\rangle}$. And $E^{\langle\rangle} \subset U_{\langle\rangle}=F$. So $(\bar{E}, \bar{g})$ satisfies (ii) in the definition of fulfillment.

We have now constructed a compatible topology, $t$, and a fulfillment, $(\bar{E}, \bar{g})$, of $F$. Let $f: \widetilde{E} \rightarrow G$ be the fulfillment function. To complete the proof of this lemma, all that remains to be shown is that $f$ is continuous with respect to $t$.

Claim. Let $\tau_{0}, \tau \in T$ be regular with $\tau_{0} \prec \tau$. Let $n_{0}=\operatorname{length}\left(\tau_{0}\right)$, and let $n=$ length $(\tau)$. Then

$$
d\left(g^{\tau_{0}}, g^{\tau}\right) \leq \sum_{k=n_{0}}^{n-1} \frac{1}{2^{k}} .
$$

Proof of Claim. The proof is by induction on $n-n_{0}$. If $n-n_{0}=0$, this is trivial. So suppose $n-n_{0}>0$. Let $\tau=\sigma^{\frown}(i)$, where $i>0$. As $\tau_{0}$ is regular, $\tau_{0} \prec \tilde{\sigma} \prec \sigma$ (see Definition 2.3.4). So the induction hypothesis tells us that

$$
d\left(g^{\tau_{0}}, g^{\tilde{\sigma}}\right) \leq \sum_{k=n_{0}}^{m(\tau)-1} \frac{1}{2^{k}},
$$

where $m(\tau)=\operatorname{length}(\tilde{\sigma})$. Recall that $g^{\tau}$ is defined to be $g^{\tilde{\sigma}} h_{\tau}$, that $h_{\tau} \in M_{m(\tau)}$, that $d$-diam $\left(M_{m(\tau)}\right) \leq 1 / 2^{m(\tau)}$ and that the metric $d$ is left-invariant. Therefore,

$$
\begin{aligned}
d\left(g^{\tilde{\sigma}}, g^{\tau}\right) & =d\left(g^{\tilde{\sigma}}, g^{\tilde{\sigma}} h_{\tau}\right) \\
& =d\left(g^{\tilde{\sigma}} e, g^{\tilde{\sigma}} h_{\tau}\right) \\
& =d\left(e, h_{\tau}\right) \\
& \leq d-\operatorname{diam}\left(M_{m(\tau)}\right) \\
& \leq \frac{1}{2^{m(\tau)}} .
\end{aligned}
$$

Clearly,

$$
\begin{aligned}
d\left(g^{\tau_{0}}, g^{\tau}\right) & \leq d\left(g^{\tau_{0}}, g^{\tilde{\sigma}}\right)+d\left(g^{\tilde{\sigma}}, g^{\tau}\right) \\
& \leq\left(\sum_{k=n_{0}}^{m(\tau)-1} \frac{1}{2^{k}}\right)+\frac{1}{2^{m(\tau)}} \\
& =\sum_{k=n_{0}}^{m(\tau)} \frac{1}{2^{k}} \\
& \leq \sum_{k=n_{0}}^{n-1} \frac{1}{2^{k}} .
\end{aligned}
$$

This proves the Claim.

To prove continuity, fix $x_{0} \in \widetilde{E}$ and fix $\varepsilon>0$. We must show that there is a $t$-open set $V \subset X$ such that $x_{0} \in V$ and such that for every $x \in(V \cap \widetilde{E})$, $d\left(f\left(x_{0}\right), f(x)\right)<\varepsilon$. 
By definition of $\widetilde{E}$, there exists a regular $\tau_{0} \in T$ such that $x_{0} \in E^{\tau_{0}}$. Since $E^{\tau_{0}}$ depends only on the equivalence class of $\tau_{0}$, we may assume that $n_{0}=\operatorname{length}\left(\tau_{0}\right)$ is large enough that $1 / 2^{\left(n_{0}-1\right)}<\varepsilon$. Let $V=V_{\tau_{0}}$. As $t$ was chosen to be finer than $t_{n_{0}}$, (10) ensures that $V$ is $t$-open. By $(2.3 .8), x_{0} \in V$. Since $x_{0} \in E^{\tau_{0}}$, by definition of the fulfillment function, $f\left(x_{0}\right)=g^{\tau_{0}}$.

Let $x \in V \cap \widetilde{E}$. Again, there exists a regular $\tau \in T$ such that $x \in E^{\tau}$, and, as above, we may assume that $n=\operatorname{length}(\tau)$ is larger than $n_{0}$. Also as above, $f(x)=g^{\tau}$. Again using (2.3.8), we see that $x \in V_{\tau}$.

Since $x \in V_{\tau} \cap V_{\tau_{0}}$, by (11) and (12), $\tau_{0} \prec \tau$. So the hypothesis of the Claim is satisfied. Therefore,

$$
\begin{aligned}
d\left(f\left(x_{0}\right), f(x)\right) & =d\left(g^{\tau_{0}}, g^{\tau}\right) \\
& \leq \sum_{k=n_{0}}^{n-1} \frac{1}{2^{k}} \\
& <\sum_{k=n_{0}}^{\infty} \frac{1}{2^{k}} \\
& =1 / 2^{\left(n_{0}-1\right)} \\
& <\varepsilon .
\end{aligned}
$$

Lemma 2.3.9. Let $D$ be a countable dense subgroup of $G$, and let $Y \subset X$ be a $\mu$-measurable set such that $\mu(Y)>0$. There exists a compatible topology, $t$, for $X$ and there exists a subset, $C$, of $Y$ such that

(a) $C$ is t-closed,

(b) $\mu(C)>0$,

(c) $\mu(D \cdot C)=\mu(G \cdot C)$.

Proof. By Theorem 1.1.4, there is a compatible topology, $\hat{t}$, for $X$. Of course, there exists a $\hat{t}$-compact subset $K$ of $Y$ such that $\mu(K)>0$.

Let $Q=D \cdot K$. By Lemma 2.1.21, for all $g \in G$ for a.e. $x \in Q, g \cdot x \in Q$. That is, $Q$ is almost invariant. Since $\mu$ is suitable, by Theorem 1.2 .8 , it is weakly suitable. By definition of weakly suitable (Definition 1.2.4), there exists an invariant (under the action by all of $G)$ Borel set $Q^{\prime}$ with $\mu\left(Q \triangle Q^{\prime}\right)=0$. Let $C=K \cap Q^{\prime}$. Clearly $C \subset K$ and $\mu(K \backslash C)=0$. Hence $\mu(C)>0$.

By Theorem 2.1.17, there exists a compatible topology, $t$, such that $t$ is finer than $\hat{t}$ and such that the invariant Borel set $X \backslash Q^{\prime}$ is $t$-open. Note that $C$ is $t$-closed.

As $D$ is countable, by quasi-invariance, $\mu(D \cdot C)=\mu(D \cdot K)$. As $Q^{\prime}$ is invariant, $G \cdot C \subset Q^{\prime}$. Therefore,

$$
\mu(G \cdot C) \leq \mu\left(Q^{\prime}\right)=\mu(Q)=\mu(D \cdot K)=\mu(D \cdot C) .
$$

Proof of Theorem 1.2.12. We have fixed a suitable measured Borel $G$-space $(X, \mu)$. Let us also fix a Borel set $A \subset X$. We must prove that there exists a compatible topology $t$, there exists a Borel $B \subset A$ with $\mu(B)=\mu(A)$ and there exists a fulfillment of $B$ whose fulfillment function is continuous with respect to $t$.

Let $D$ be a countable dense subgroup of $G$. Call a set $C \subset X$ nice if it satisfies all four of the following conditions.

(a) There exists a compatible topology, $t^{C}$, such that $C$ is $t^{C}$-closed.

(b) $\mu(C)>0$. 
(c) $\mu(D \cdot C)=\mu(G \cdot C)$.

(d) $C \subset A$.

Let $\mathcal{F}$ be a maximal family of nice sets with the property that

$$
\text { for all } C_{1}, C_{2} \in \mathcal{F} \text {, if } C_{1} \neq C_{2} \text {, then } G \cdot C_{1} \cap G \cdot C_{2}=\emptyset \text {. }
$$

Let $F=\bigcup \mathcal{F}$. Clearly such an $\mathcal{F}$ exists and is countable, and clearly $F$ is a Borel subset of $A$.

Now $(G \cdot F) \cap A$ is an analytic set, hence measurable, so there is a Borel set $B$ with $F \subset B \subset((G \cdot F) \cap A)$ such that $\mu(((G \cdot F) \cap A) \backslash B)=0$. Since $A \backslash(G \cdot F)$ is coanalytic, it is measurable. So by maximality and Lemma 2.3.9, $\mu(A \backslash(G \cdot F))=0$. Therefore, $\mu(B)=\mu(A)$.

Since $F \subset B \subset G \cdot F$, any fulfillment of $F$ is also a fulfillment of $B$. So in order to prove this theorem, it will suffice to construct a fulfillment of $F$-rather than of $B$-with the required continuity property. In light of Lemma 2.3.7, in order to do this we need only show the following two facts. First, $\mu(D \cdot F)=\mu(G \cdot F)$. Second, there exists a compatible topology, $\hat{t}$, for $X$ such that $F$ is $\hat{t}$-closed.

The first of these facts follows from (c) in the definition of nice.

To prove the second fact, for each $C \in \mathcal{F}$, fix a compatible topology $t^{C}$ satisfying (a) in the definition of nice. Let $\mathcal{F}^{*}=\{G \cdot C: C \in \mathcal{F}\}$. The members of $\mathcal{F}^{*}$ are invariant analytic sets and, by (2.3.10), they are pairwise disjoint. By Lemma 2.1.16, any two members of $\mathcal{F}^{*}$ can be separated by an invariant Borel set; taking countable intersections, we obtain a family $\left\{B^{C}: C \in \mathcal{F}\right\}$ of pairwise disjoint invariant Borel sets such that for all $C \in \mathcal{F}, G \cdot C \subset B^{C}$. By Theorem 2.1.17, for each $C \in \mathcal{F}$, there exists a compatible topology $\tilde{t}^{C}$ such that $B^{C}$ is $\tilde{t}^{C}$-open; and there exists a compatible topology $\tilde{t}^{*}$ such that $\left(X \backslash \bigcup\left\{B^{C}: C \in \mathcal{F}\right\}\right)$ is $\tilde{t}^{*}$-open. Then Corollary 2.1.18 gives us a compatible topology, $\hat{t}$, for $X$ such that $\hat{t}$ is finer than each $t^{C}$, finer than each $\tilde{t}^{C}$ and finer than $\tilde{t}^{*}$. Clearly $F$ is $\hat{t}$-closed.

2.4. Proof of Theorem A. Throughout $§ 2.4$, we have fixed Polish groups $G$ and $H$ and a Polish $G$-space, $X$.

We begin $\S 2.4$ by considering the problem of extending functions satisfying the cocycle identity from subsets of $G \times X$ to all of $G \times X$. In this part of $\S 2.4$, there are no measures, hence no almost cocycle - only strict cocycles.

Definition 2.4.1. $\quad$ (a) A set $P \subset X$ is Bairely invariant if for all $x \in P$, for comeager many $g \in G, g \cdot x \in P$.

(b) A set $S \subset G \times X$ is called sufficiently closed if it satisfies the following two closure properties.

(i) For all $g_{1}, g_{2} \in G$, for all $x \in X$, if $\left(g_{2}, x\right) \in S$ and $\left(g_{1}, g_{2} \cdot x\right) \in S$, then $\left(g_{1} g_{2}, x\right) \in S$

(ii) For all $g \in G$, for all $x \in X$, if $(g, x) \in S$, then $\left(g^{-1}, g \cdot x\right) \in S$.

Recall the definition of $Q(P)$ (Definition 2.1.14).

Lemma 2.4.2. (a) For any set $P \subset X, Q(P)$ is sufficiently closed.

(b) For any set $S \subset G \times X$, if $S$ is sufficiently closed and

$$
P=\{x \in X: \text { for comeager many } g \in G,(g, x) \in S\},
$$

then $P$ is Bairely invariant and $Q(P) \subset S$. 
Proof. (a) Obvious.

(b) To prove that $P$ is Bairely invariant, it will suffice to show that if $x \in P$ and $(k, x) \in S$, then $k \cdot x \in P$. Fix such an $x$ and $k$. Part (ii) of the definition of sufficiently closed implies that $\left(k^{-1}, k \cdot x\right) \in S$. So by part (i) of that definition, for every $g \in G$, if $(g, x) \in S$, that is, if $\left(g, k^{-1} \cdot(k \cdot x)\right) \in S$, then $\left(g k^{-1}, k \cdot x\right) \in S$. Thus

$$
\{g:(g, x) \in S\} k^{-1} \subset\left\{g k^{-1}:\left(g k^{-1}, k \cdot x\right) \in S\right\} .
$$

Since the smaller set is comeager, so is the larger set.

To prove that $Q(P) \subset S$, we must show that for all $x, y \in P$ and for all $k \in G$, if $k \cdot x=y$, then $(k, x) \in S$. So fix $x, y$ and $k$. By definition of $P$, there is a $g \in G$ such that $(g k, x) \in S$ and $(g, y) \in S$. As $y=k \cdot x,(g, k \cdot x) \in S$. Again using both parts of the definition of sufficiently closed: $\left(g^{-1}, g k \cdot x\right) \in S$; hence $\left(g^{-1}(g k), x\right) \in S$. So $(k, x) \in S$.

Definition 2.4.3. A partial cocycle is a pair $(Q, f)$ where:

(a) $Q$ is a sufficiently closed Borel subset of $G \times X$;

(b) $f: Q \rightarrow H$ is a Borel-measurable function and for all $g_{1}, g_{2} \in G$ and for all $x \in X$, if $\left(g_{2}, x\right) \in Q$ and $\left(g_{1}, g_{2} \cdot x\right) \in Q$, then $f\left(g_{1} g_{2}, x\right)=$ $f\left(g_{1}, g_{2} \cdot x\right) f\left(g_{2}, x\right)$.

The next lemma is the Baire-category analog of a measure theoretic lemma of Mackey, a lemma which is a part of his proof of the Mackey Cocycle Theorem (Theorem 1.3.11).

Lemma 2.4.4. Let $P$ be a Bairely invariant Borel subset of $X$. Suppose that $(Q(P), f)$ is a partial cocycle. Then there exists a strict cocycle $\varphi: G \times X \rightarrow H$ such that $\varphi\lceil Q(P)=f$.

Proof. As $P$ is Bairely invariant,

$$
G \cdot P=\{x \in X: \text { for comeager many } g \in G, g \cdot x \in P\} .
$$

This fact has two consequences. First, by Lemma 2.1.1(a), $G \cdot P$ is Borel. Second, the Borel relation

$$
R=\{(x, g) \in X \times G: x \in G \cdot P \& g \cdot x \in P\}
$$

has the property that for every $x \in \operatorname{dom}(R), R_{x}$ is comeager. By Lemma 2.1.2, $R$ has a Borel-measurable uniformizing function $\hat{\theta}: G \cdot P \rightarrow G$, i.e., for all $x \in G \cdot P$, $\hat{\theta}(x) \cdot x \in P$. We now modify $\hat{\theta}$ to produce $\theta: G \cdot P \rightarrow G$, by defining

$$
\theta(x)= \begin{cases}\hat{\theta}(x), & \text { if } \quad x \notin P, \\ e, & \text { if } \quad x \in P .\end{cases}
$$

Since $P$ is Borel, $\theta$ is Borel-measurable, and, of course, $\theta$ is still a uniformizing function for $R$.

Let $x \in G \cdot P$ and $g \in G$, and consider the following element of $G$ : $\theta(g \cdot x) g(\theta(x))^{-1}$. Note that $\theta(x) \cdot x$ and $\theta(g \cdot x) \cdot(g \cdot x)$ are in $P$, and

$$
\left[\theta(g \cdot x) g(\theta(x))^{-1}\right] \cdot[\theta(x) \cdot x]=[\theta(g \cdot x) \cdot(g \cdot x)] .
$$

So by definition of $Q(P)$,

$$
\left(\theta(g \cdot x) g(\theta(x))^{-1}, \theta(x) \cdot x\right) \in Q(P)=\operatorname{dom}(f) .
$$


Therefore, the following function $\varphi: G \times X \rightarrow H$ is well defined:

$$
\varphi(g, x)=\left\{\begin{array}{l}
f\left(\theta(g \cdot x) g(\theta(x))^{-1}, \theta(x) \cdot x\right), \text { if } x \in G \cdot P, \\
e, \text { if } x \notin G \cdot P .
\end{array}\right.
$$

Since $G \cdot P$ is a Borel set and $f, \theta$ and the action are Borel-measurable functions, $\varphi$ is also Borel-measurable.

Obviously, $\varphi$ satisfies the cocycle identity on the invariant set $X \backslash(G \cdot P)$. To prove the cocycle identity on $G \cdot P$, let $x \in G \cdot P$ and let $g_{1}, g_{2} \in G$. Since $f$ satisfies the cocycle identity, one can easily check that

$$
\begin{aligned}
& \varphi\left(g_{1} g_{2}, x\right) \\
&= f\left[\theta\left(g_{1} g_{2} \cdot x\right) g_{1} g_{2}(\theta(x))^{-1}, \theta(x) \cdot x\right] \\
&= f\left[\left[\theta\left(g_{1} \cdot\left(g_{2} \cdot x\right)\right) g_{1}\left(\theta\left(g_{2} \cdot x\right)\right)^{-1}\right]\left[\theta\left(g_{2} \cdot x\right) g_{2}(\theta(x))^{-1}\right], \theta(x) \cdot x\right] \\
&= {\left[f\left[\theta\left(g_{1} \cdot\left(g_{2} \cdot x\right)\right) g_{1}\left(\theta\left(g_{2} \cdot x\right)\right)^{-1},\left(\theta\left(g_{2} \cdot x\right) g_{2}(\theta(x))^{-1}\right) \cdot(\theta(x) \cdot x)\right]\right] } \\
& {\left[f\left[\theta\left(g_{2} \cdot x\right) g_{2}(\theta(x))^{-1}, \theta(x) \cdot x\right]\right] } \\
&= {\left[f\left[\theta\left(g_{1} \cdot\left(g_{2} \cdot x\right)\right) g_{1}\left(\theta\left(g_{2} \cdot x\right)\right)^{-1}, \theta\left(g_{2} \cdot x\right) \cdot\left(g_{2} \cdot x\right)\right]\right] } \\
& {\left[f\left[\theta\left(g_{2} \cdot x\right) g_{2}(\theta(x))^{-1}, \theta(x) \cdot x\right]\right] } \\
&= \varphi\left(g_{1}, g_{2} \cdot x\right) \varphi\left(g_{2}, x\right) .
\end{aligned}
$$

Finally, to show that $\varphi\lceil Q(P)=f$, let $(g, x) \in Q(P)$. Then both $x$ and $g \cdot x$ are in $P$, so $\theta(x)=\theta(g \cdot x)=e$. Therefore,

$$
\begin{aligned}
\varphi(g, x) & =f\left(\theta(g \cdot x) g(\theta(x))^{-1}, \theta(x) \cdot x\right) \\
& =f\left(e g e^{-1}, e \cdot x\right) \\
& =f(g, x) .
\end{aligned}
$$

Definition 2.4.5. A framework is a triple $(D, E, F)$ satisfying the following four properties:

(a) $D$ is a countable dense subgroup of $G$.

(b) $E$ is a Borel subset of $X$ which is invariant under the action by $D$

(c) $(D \times E, F)$ is a partial cocycle.

(d) $F: D \times E \rightarrow H$ is continuous, where $D$ and $E$ are topologized as subspaces of $G$ and $X$, respectively.

Note that parts (a) and (b) of Definition 2.4.5 imply that $D \times E$ is sufficiently closed; so part (c) merely states that $F$ is a function from $D \times E$ into $H$ which satisfies the cocycle identity. Using Zorn's Lemma, $F$ can be extended to a function $\widehat{F}: G \times X \rightarrow H$ satisfying the cocycle identity-but such an $\widehat{F}$ will not be Borelmeasurable. The next lemma states that, in fact, we can extend $F$ in a Borel way.

Lemma 2.4.6. Let $(D, E, F)$ be a framework. There exists a strict cocycle $\varphi$ such that $\varphi\lceil(D \times E)=F$.

Proof. Let $d$ be a metric on $H$. By definition of framework, $F: D \times E \rightarrow H$ is continuous. Let $S \subset G \times X$ and $\widetilde{F}: S \rightarrow H$ be the canonical continuous extension 
of $F$ (with respect to $d$ ) to a maximal $G_{\delta}$ domain (see Lemma 2.1.3); that is, $S=\bigcap\left\{S_{\varepsilon}: \varepsilon>0\right\}$ where

$S_{\varepsilon}=\{(g, x)$ : there exists an open nbhd $N$ of $G \times X$ with $(g, x) \in N$ such that

for all points $(k, y)$ and $\left(k^{\prime}, y^{\prime}\right)$ in $\left.N \cap(D \times E), d\left(F(k, y), F\left(k^{\prime}, y^{\prime}\right)\right)<\varepsilon\right\}$.

Claim. $S$ is sufficiently closed.

Proof of Claim. To prove part (i) of the definition of sufficiently closed (Definition 2.4.1 (b)), fix $g_{1}, g_{2} \in G$ and $x \in X$ such that $\left(g_{2}, x\right) \in S$ and $\left(g_{1}, g_{2} \cdot x\right) \in S$, and fix $\varepsilon>0$. We must show that there is a neighborhood $N$ of $\left(g_{1} g_{2}, x\right)$ witnessing that $\left(g_{1} g_{2}, x\right) \in S_{\varepsilon}$. Let $M_{1}$ and $M_{2}$ be open neighborhoods of $H$, containing $\widetilde{F}\left(g_{1}, g_{2} \cdot x\right)$ and $\widetilde{F}\left(g_{2}, x\right)$, respectively, such that $d$-diam $\left(M_{1} M_{2}\right)<\varepsilon$. By definition of $S$, there exist neighborhoods $N_{1}^{G}, N_{2}^{G}, N_{1}^{X}$, and $N_{2}^{X}$ of $G, G, X$, and $X$, respectively, containing $g_{1}, g_{2}, g_{2} \cdot x$, and $x$, respectively, and such that

$$
\begin{aligned}
& F\left[\left(N_{1}^{G} \times N_{1}^{X}\right) \cap(D \times E)\right] \subset M_{1} \text { and } \\
& F\left[\left(N_{2}^{G} \times N_{2}^{X}\right) \cap(D \times E)\right] \subset M_{2} .
\end{aligned}
$$

Since the action is continuous, without loss of generality, $N_{2}^{G} \cdot N_{2}^{X} \subset N_{1}^{X}$. Then

$$
N=N_{1}^{G} N_{2}^{G} \times N_{2}^{X}
$$

is a neighborhood of $\left(g_{1} g_{2}, x\right)$. To prove that this $N$ works, we need only show that if $(k, y) \in N \cap(D \times E)$, then $F(k, y) \in M_{1} M_{2}$. This is straightforward, using the fact that - since $D$ is a dense subgroup of $G$ - there are $k_{1} \in N_{1}^{G} \cap D$ and $k_{2} \in N_{2}^{G} \cap D$ such that $k=k_{1} k_{2}$, and the fact that $F$ satisfies the cocycle identity. To prove part (ii) of the definition of sufficiently closed, fix $\left(g_{0}, x_{0}\right) \in S$ and $\varepsilon>0$. Let $M$ be an open neighborhood of $H$ containing $\widetilde{F}\left(g_{0}, x_{0}\right)$ such that $d$-diam $\left(M^{-1}\right)<\varepsilon$. By definition of $S$, there is an open neighborhood $N_{0}$ of $\left(g_{0}, x_{0}\right)$ such that $F\left[N_{0} \cap(D \times E)\right] \subset M$. The function $\mathcal{H}:(g, x) \mapsto\left(g^{-1}, g \cdot x\right)$ is a homeomorphism of $G \times X$ onto itself. So $N=\mathcal{H}\left[N_{0}\right]$ is an open neighborhood of $\left(g_{0}^{-1}, g_{0} \cdot x_{0}\right)$. By Proposition 2.1.22(a)(ii), $F\left(k^{-1}, k \cdot y\right)=(F(k, y))^{-1}$, which implies that the neighborhood $N$ witnesses that $\left(g_{0}^{-1}, g_{0} \cdot x_{0}\right) \in S_{\varepsilon}$. This proves the Claim.

Let

$$
P=\{x \in X: \text { for comeager many } g \in G,(g, x) \in S\} .
$$

By Lemma 2.4.2, $P$ is Bairely invariant, $Q(P) \subset S$ and $Q(P)$ is sufficiently closed. Since $S$ is $G_{\delta}$, by Lemma 2.1.1(b), $P$ is also $G_{\delta}$, hence Borel; so by definition of $Q(P)$ (Definition 2.1.14), $Q(P)$ is Borel. Let $f=\widetilde{F}\lceil Q(P)$. By continuity, the cocycle identity extends from $F$ to $\widetilde{F}$. Thus $f$ is continuous, hence Borel, and satisfies the cocycle identity. We have therefore shown that $(Q(P), f)$ is a partial cocycle. Lemma 2.4.4 now gives us a strict cocycle $\varphi: G \times X \rightarrow H$ such that $\varphi\lceil Q(P)=f$.

Consider a point $x \in E$. Since $S$ is a $G_{\delta}$ subset of $G \times X, S^{x}$ is a $G_{\delta}$ subset of $G$. Clearly

$$
D \times\{x\} \subset D \times E \subset S,
$$

so $D \subset S^{x}$. As $D$ is dense, $S^{x}$ is comeager. So by definition of $P, x \in P$. This shows that $E \subset P$. 
By definition of framework, $E$ is invariant under the action by $D$. So for all $(g, x) \in D \times E$, both $x$ and $g \cdot x$ are in $P$. By definition of $Q(P)$, this means that $D \times E \subset Q(P)$. Therefore,

$$
\varphi\lceil(D \times E)=f\lceil(D \times E)=\widetilde{F}\lceil(D \times E)=F .
$$

Lemma 2.4.6, above, completes our consideration of extending functions satisfying the cocycle identity. We next turn our attention to almost cocycles rather than strict cocycles. For the rest of $\S 2.4$, we have a fixed quasi-invariant Borel probability measure, $\mu$, on the Polish $G$-space $X$.

Lemma 2.4.7. Let $d$ be a right-invariant metric on $H$ with finite diameter, and let $\psi: G \times X \rightarrow H$ be an almost cocycle. For any sequence $g_{n}$ of elements of $G$ and for any $g \in G$, if $g_{n}$ converges to $g$, then $\psi_{g_{n}}$ converges to $\psi_{g}$ in measure (with respect to $\mu$ and $d$ ).

Proof. Let $C$ be a comeager subset of $G$ which satisfies Lemma 2.1.9 (for $W=G$, $Y=H$ and $F=\psi$ ).

To prove this lemma, consider fixed elements $g_{n}$ and $g$ of $G$ such that $g_{n}$ converges to $g$. Since $C$ is comeager, there is a $k \in G$ such that $g k \in C$ and for all $n \in \mathbb{N}$, $g_{n} k \in C$. Fix such a $k$. Then

$$
\psi_{g_{n} k} \text { converges in measure to } \psi_{g k} \text {. }
$$

Let $\hat{g}$ denote either $g$ or one of the $g_{n}$ 's. As $\psi$ is an almost cocycle, for almost every $x \in X$,

$$
\psi(\hat{g}, k \cdot x)=\psi(\hat{g} k, x)[\psi(k, x)]^{-1} .
$$

Let $Y$ be a measure 0 subset of $X$ such that for $x \in X \backslash Y$, the above equality holds for all such $\hat{g}$. Using the right-invariance of $d$, we see that for all $n \in \mathbb{N}$ and for all $x \in X \backslash Y$,

$$
\begin{aligned}
& d\left(\psi\left(g_{n}, k \cdot x\right), \psi(g, k \cdot x)\right) \\
& \quad=d\left(\psi\left(g_{n} k, x\right)[\psi(k, x)]^{-1}, \psi(g k, x)[\psi(k, x)]^{-1}\right) \\
& \quad=d\left(\psi\left(g_{n} k, x\right), \psi(g k, x)\right) .
\end{aligned}
$$

For all $n \in \mathbb{N}$ and all $\varepsilon>0$, define

$$
\begin{aligned}
& A_{n}^{\varepsilon}=\left\{x \in X: d\left(\psi\left(g_{n} k, x\right), \psi(g k, x)\right) \geq \varepsilon\right\}, \\
& B_{n}^{\varepsilon}=\left\{x^{\prime} \in X: d\left(\psi\left(g_{n}, x^{\prime}\right), \psi\left(g, x^{\prime}\right)\right) \geq \varepsilon\right\} .
\end{aligned}
$$

Note that

$$
\begin{aligned}
B_{n}^{\varepsilon} \backslash k & \cdot Y \\
& =\left\{k \cdot x \in X: d\left(\psi\left(g_{n}, k \cdot x\right), \psi(g, k \cdot x)\right) \geq \varepsilon\right\} \backslash k \cdot Y \\
& =\left\{k \cdot x \in X: d\left(\psi\left(g_{n} k, x\right), \psi(g k, x)\right) \geq \varepsilon\right\} \backslash k \cdot Y \\
& =k \cdot A_{n}^{\varepsilon} \backslash k \cdot Y .
\end{aligned}
$$

So $B_{n}^{\varepsilon} \subset\left(k \cdot A_{n}^{\varepsilon} \cup k \cdot Y\right)$.

By (2.4.8) and the definitions of $A_{n}^{\varepsilon}$ and of convergence in measure (Definition 2.1.8), for all $\varepsilon>0, \mu\left(A_{n}^{\varepsilon}\right)$ converges to 0 . Therefore, by Lemma 2.1.20, for all $\varepsilon>0, \mu\left(k \cdot A_{n}^{\varepsilon}\right)$ also converges to 0 . So for all $\varepsilon>0, \mu\left(B_{n}^{\varepsilon}\right)$ converges to 0 . This means, by definition of $B_{n}^{\varepsilon}$ and of convergence in measure, that $\psi_{g_{n}}$ converges to $\psi_{g}$ in measure. 
Lemma 2.4.9. Let $\varphi$ and $\psi$ be two almost cocycles. Suppose that there exists a dense subset $D$ of $G$ and a subset $E$ of $X$ with $\mu(E)=1$ such that $\varphi\lceil(D \times E)=$ $\psi\lceil(D \times E)$. Then $\varphi$ and $\psi$ are equivalent.

Proof. By Theorem 2.1.11, there exists a right-invariant metric $d$ on $H$ with finite diameter. Given such a $d$, apply Lemma 2.4 .7 to both $\varphi$ and $\psi$.

Proof of Theorem A. We have fixed Polish groups $G$ and $H$ and a measured Polish $G$-space $(X, \mu)$. Let us also fix a Borel-measurable almost cocycle $\psi: G \times X \rightarrow H$ such that $\psi$ is essentially continuous. We must prove that there exists a Borelmeasurable strict cocycle $\varphi: G \times X \rightarrow H$ which is equivalent to $\psi$.

By definition of essential continuity (Definition 1.4.1(a)), there exists a Borel set $S \subset G \times X$ satisfying the following two conditions:

(i) For all $g \in G$, for a.e. $x \in X,(g, x) \in S$.

(ii) $\psi\lceil S$ is continuous.

Fix a countable dense subgroup, $D$, of $G$. Since $D$ is countable, we can - using (i), quasi-invariance, and the fact that $\psi$ is an almost cocycle - obtain a Borel set $E \subset X$ such that

(a) $\mu(E)=1$,

(b) for all $g \in D, E \subset S_{g}$,

(c) for all $g \in D$, for all $x \in E, g \cdot x \in E$,

(d) for all $g_{1}, g_{2} \in D$, for all $x \in E, \psi\left(g_{1} g_{2}, x\right)=\psi\left(g_{1}, g_{2} \cdot x\right) \psi\left(g_{2}, x\right)$.

Now (ii) and (b) imply that $\psi\lceil(D \times E)$ is continuous. This fact, together with (c) and (d), means that $(D, E, \psi\lceil(D \times E))$ is a framework (see Definition 2.4.5). Applying Lemma 2.4.6 to this framework, we obtain a strict cocycle $\varphi$ such that $\varphi\lceil(D \times E)=\psi\lceil(D \times E)$. The equivalence of $\varphi$ and $\psi$ follows from Lemma 2.4.9.

2.5. Proof of Theorem B. Let $\varphi$ be a Borel-measurable strict cocycle on the measured Polish $G$-space $(X, \mu)$ with values in $H$. We must prove that $\varphi$ is nearly essentially continuous.

Fix $\varepsilon>0$. By Lemma 2.1.6, there exists a compact $K \subset X$ such that $\mu(K)>$ $1-\varepsilon$ and there exists a Borel set $B \subset G \times K$ satisfying the following two properties.

(a) For all $x \in K$, for comeager many $g \in G,(g, x) \in B$.

(b) $\varphi\lceil B$ is continuous.

Let $S=\{(g, x) \in G \times X: x \in K$ and $g \cdot x \in K\}$. It is trivial that $K$ and $S$ satisfy condition (i) in the definition of near essential continuity (Definition 1.4.1(b)). So to complete the proof of this theorem, all that remains to be shown is that condition (ii) of that definition is satisfied: $\varphi\lceil S$ is continuous. To prove this, fix a point $\left(g_{0}, x_{0}\right) \in S$ and fix an open neighborhood $M_{0}$ of the target group, $H$, containing $\varphi\left(g_{0}, x_{0}\right)$. We must find open neighborhoods $N_{0}^{G}$ and $N_{0}^{X}$ of $G$ and $X$, respectively, containing $g_{0}$ and $x_{0}$, respectively, such that

$$
\varphi\left[\left(N_{0}^{G} \times N_{0}^{X}\right) \cap S\right] \subset M_{0} .
$$

As both $x_{0}$ and $g_{0} \cdot x_{0}$ are in $K$, it follows from (a) that there is a $k \in G$ such that $\left(k, g_{0} \cdot x_{0}\right) \in B$ and $\left(k g_{0}, x_{0}\right) \in B$. Since $\varphi$ is a strict cocycle,

$$
\varphi\left(g_{0}, x_{0}\right)=\left[\varphi\left(k, g_{0} \cdot x_{0}\right)\right]^{-1} \varphi\left(k g_{0}, x_{0}\right) .
$$

Let $M_{1}$ and $M_{2}$ be open neighborhoods of the target group containing $\varphi\left(k, g_{0} \cdot x_{0}\right)$ and $\varphi\left(k g_{0}, x_{0}\right)$, respectively, and such that $M_{1}^{-1} M_{2} \subset M_{0}$. Now (b) tells us that 
there exist neighborhoods $N_{1}^{G}, N_{2}^{G}, N_{1}^{X}$, and $N_{2}^{X}$ of $G, G, X$, and $X$, respectively, containing $k, k g_{0}, g_{0} \cdot x_{0}$, and $x_{0}$, respectively, such that

(i) $\quad \varphi\left[\left(N_{1}^{G} \times N_{1}^{X}\right) \cap B\right] \subset M_{1}$,

(ii) $\quad \varphi\left[\left(N_{2}^{G} \times N_{2}^{X}\right) \cap B\right] \subset M_{2}$.

Furthermore, $N_{1}^{G}$ can be chosen so that there is an open neighborhood $\widetilde{N}$ of $g_{0}$ with $N_{1}^{G} \tilde{N} \subset N_{2}^{G}$. Since the action is continuous, there are open neighborhoods $N_{0}^{G}$ and $N_{0}^{X}$ of $G$ and $X$, respectively, containing $g_{0}$ and $x_{0}$, respectively, such that

$$
N_{1}^{G} N_{0}^{G} \subset N_{2}^{G}
$$$$
\text { (iii) } \quad N_{0}^{G} \cdot N_{0}^{X} \subset N_{1}^{X} \text {. }
$$

Now let $\left(g_{2}, x\right)$ be an arbitrary point in $\left[\left(N_{0}^{G} \times N_{0}^{X}\right) \cap S\right]$. We show that $\varphi\left(g_{2}, x\right) \in$ $M_{0}$. By definition of $S$, both $x$ and $g_{2} \cdot x$ are in $K$, so, again, it follows from (a) that there is a $g_{1} \in N_{1}^{G}$ such that $\left(g_{1}, g_{2} \cdot x\right) \in B$ and $\left(g_{1} g_{2}, x\right) \in B$. By (2.5.2)(iii), $g_{2} \cdot x \in N_{1}^{X}$, so (2.5.1)(i) implies that $\varphi\left(g_{1}, g_{2} \cdot x\right) \in M_{1}$. We now use the other parts of (2.5.2): by (i), $g_{1} g_{2} \in N_{2}^{G}$, and by (ii), $x \in N_{2}^{X}$. So (2.5.1)(ii) implies that $\varphi\left(g_{1} g_{2}, x\right) \in M_{2}$. Again using the fact that $\varphi$ is a strict cocycle, we see that

$$
\varphi\left(g_{2}, x\right)=\left[\varphi\left(g_{1}, g_{2} \cdot x\right)\right]^{-1} \varphi\left(g_{1} g_{2}, x\right) \in M_{1}^{-1} M_{2} \subset M_{0} .
$$

2.6. Proof of Theorem C. Throughout $\S 2.6$, all of the following are fixed: Polish groups $G$ and $H$; a suitable measured Borel $G$-space $(X, \mu)$; and a Borel-measurable almost cocycle $\psi: G \times X \rightarrow H$. We consider many different compatible topologies for $X$.

Recall the definitions of fulfillment and of the fulfillment function Definition 1.2.10.

Definition 2.6.1. Let $B \subset X$ be a Borel set such that $\mu(G \cdot B)=1$, and let $(\bar{E}, \bar{g})$ be a fulfillment of $B$ with fulfillment function $f: \widetilde{E} \rightarrow G$.

(a) The extended fulfilment function $\hat{f}: X \rightarrow G$ is defined as follows:

$$
\hat{f}(x)= \begin{cases}f(x), & \text { if } x \in \widetilde{E} \\ e, & \text { if } x \notin \widetilde{E} .\end{cases}
$$

(b) We define a new function $\varphi: G \times X \rightarrow H$, called the fulfilled cocycle associated with $(\psi, B, \bar{E}, \bar{g})$, as follows:

$$
\varphi(g, x)=\psi\left(\hat{f}(g \cdot x) g(\hat{f}(x))^{-1}, \hat{f}(x) \cdot x\right) .
$$

In the first part of $\$ 2.6$ - through Lemma 2.6.5 - we consider properties of the fulfilled cocycle. The terminology is justified by the following lemma, which states that $\varphi$ is, in fact, an almost cocycle, that is, a Borel-measurable almost cocycle on the measured $G$-space $(X, \mu)$ with values in $H$.

Lemma 2.6.2. Let $B \subset X$ be a Borel set such that $\mu(G \cdot B)=1$, and let $(\bar{E}, \bar{g})$ be a fulfilment of $B$. Let $\varphi$ be the fulfilled cocycle associated with $(\psi, B, \bar{E}, \bar{g})$.

(a) $\varphi$ is Borel-measurable.

(b) For all $k_{1}, k_{2} \in G$, for a.e. $x \in X, \varphi\left(k_{1} k_{2}, x\right)=\varphi\left(k_{1}, k_{2} \cdot x\right) \varphi\left(k_{2}, x\right)$. 
Proof. Let $\bar{E}=E_{0}, E_{1}, E_{2}, \ldots$, let $\bar{g}=g_{0}, g_{1}, g_{2}, \ldots$, let $\widetilde{E}=\bigcup_{i} E_{i}$, let $f: \widetilde{E} \rightarrow G$ be the fulfillment function, and let $\hat{f}: X \rightarrow G$ be the extended fulfillment function.

(a) It will suffice to show that $\hat{f}$ is Borel-measurable. By definition of $\hat{f}$ and $f, \hat{f}$ is constant on each of the Borel sets $E_{i}$ and $X \backslash\left(\bigcup_{i} E_{i}\right)$.

(b) Suppose the cocycle identity is false: fix $k_{1}, k_{2} \in G$ and fix a Borel set $D \subset X$ such that $\mu(D)>0$ and for all $x \in D$,

$$
\varphi\left(k_{1} k_{2}, x\right) \neq \varphi\left(k_{1}, k_{2} \cdot x\right) \varphi\left(k_{2}, x\right) .
$$

Since the measure is quasi-invariant, by definition of fulfillment, there exist natural numbers $m, n$ and $p$ such that if we set

$$
\widehat{D}=\left\{x \in D: x \in E_{m} \& k_{2} \cdot x \in E_{n} \& k_{1} k_{2} \cdot x \in E_{p}\right\},
$$

then $\mu(\widehat{D})>0$. Using the definitions of $\varphi, f$ and $\widehat{D}$ and the facts that $\mu$ is quasiinvariant and that $\psi$ is an almost cocycle, one can see that all of the following equations hold for a.e. $x \in \widehat{D}$ :

$$
\begin{aligned}
\varphi & \left(k_{1} k_{2}, x\right) \\
= & \psi\left(f\left(k_{1} k_{2} \cdot x\right) k_{1} k_{2}(f(x))^{-1}, f(x) \cdot x\right) \\
= & \psi\left(g_{p} k_{1} k_{2} g_{m}^{-1}, g_{m} \cdot x\right) \\
= & \psi\left(g_{p} k_{1} g_{n}^{-1} g_{n} k_{2} g_{m}^{-1}, g_{m} \cdot x\right) \\
= & \psi\left(g_{p} k_{1} g_{n}^{-1}, g_{n} k_{2} g_{m}^{-1} \cdot\left(g_{m} \cdot x\right)\right) \psi\left(g_{n} k_{2} g_{m}^{-1}, g_{m} \cdot x\right) \\
= & \psi\left(g_{p} k_{1} g_{n}^{-1}, g_{n} \cdot\left(k_{2} \cdot x\right)\right) \psi\left(g_{n} k_{2} g_{m}^{-1}, g_{m} \cdot x\right) \\
= & \psi\left(f\left(k_{1} k_{2} \cdot x\right) k_{1}\left(f\left(k_{2} \cdot x\right)\right)^{-1}, f\left(k_{2} \cdot x\right) \cdot\left(k_{2} \cdot x\right)\right) \\
& \psi\left(f\left(k_{2} \cdot x\right) k_{2}(f(x))^{-1}, f(x) \cdot x\right) \\
= & \varphi\left(k_{1}, k_{2} \cdot x\right) \varphi\left(k_{2}, x\right) .
\end{aligned}
$$

Lemma 2.6.3. Let $B \subset X$ be a Borel set such that $\mu(G \cdot B)=1$, and let $(\bar{E}, \bar{g})$ be a fulfilment of $B$. Let $\varphi$ be the fulfilled cocycle associated with $(\psi, B, \bar{E}, \bar{g})$. Then $\varphi$ is cohomologous to $\psi$.

Proof. Let $\bar{E}=E_{0}, E_{1}, E_{2}, \ldots$, let $\bar{g}=g_{0}, g_{1}, g_{2}, \ldots$, let $\widetilde{E}=\bigcup_{i} E_{i}$, let $f: \widetilde{E} \rightarrow G$ be the fulfillment function, and let $\hat{f}: X \rightarrow G$ be the extended fulfillment function. Define $F: X \rightarrow H$ to be the function

$$
F(x)=\psi(\hat{f}(x), x) .
$$

As in the proof of part (a) of Lemma 2.6.2, $F$ is Borel-measurable. We prove that $F$ witnesses the definition of cohomologous (Definition 1.3.6). Assume, towards a contradiction, that this is not so: fix a $k \in G$ and a Borel set $D \subset X$ such that $\mu(D)>0$ and for all $x \in D$,

$$
\varphi(k, x) \neq F(k \cdot x) \psi(k, x)[F(x)]^{-1} .
$$

We follow the pattern of the proof of Lemma 2.6.2(b): there exist natural numbers $m$ and $n$ such that if we set

$$
\widehat{D}=\left\{x \in D: x \in E_{m} \& k \cdot x \in E_{n}\right\},
$$

then $\mu(\widehat{D})>0$. Using the definitions of $\varphi, f, \widehat{D}$ and $F$, the facts that $\mu$ is quasiinvariant and that $\psi$ is an almost cocycle, and part (b)(ii) of Proposition 2.1.22, 
one can see that all of the following equations hold for a.e. $x \in \widehat{D}$ :

$$
\begin{aligned}
\varphi(k, x) & =\psi\left(f(k \cdot x) k(f(x))^{-1}, f(x) \cdot x\right) \\
& =\psi\left(g_{n} k g_{m}^{-1}, g_{m} \cdot x\right) \\
& =\psi\left(g_{n} k, g_{m}^{-1} g_{m} \cdot x\right) \psi\left(g_{m}^{-1}, g_{m} \cdot x\right) \\
& =\psi\left(g_{n} k, x\right) \psi\left(g_{m}^{-1}, g_{m} \cdot x\right) \\
& =\psi\left(g_{n} k, x\right)\left[\psi\left(g_{m}, x\right)\right]^{-1} \\
& =\psi\left(g_{n}, k \cdot x\right) \psi(k, x)\left[\psi\left(g_{m}, x\right)\right]^{-1} \\
& =\psi(f(k \cdot x), k \cdot x) \psi(k, x)[\psi(f(x), x)]^{-1} \\
& =F(k \cdot x) \psi(k, x)[F(x)]^{-1} .
\end{aligned}
$$

The next definition is a variant of Definition 1.4.1. Recall the definition of $Q(P)$ (Definition 2.1.14).

Definition 2.6.4. Suppose that $X$ is a Polish $G$-space, and let $B \subset X$ be a Borel set. $\psi$ is called B-essentially continuous if there exists a Borel set $T \subset G \times X$ satisfying the following two conditions:

(a) For all $g \in G$, for a.e. $x \in X$, if $(g, x) \in Q(B)$, then $(g, x) \in T$.

(b) $\psi\lceil T$ is continuous.

Lemma 2.6.5. Suppose that $X$ is a Polish $G$-space. Let $B \subset X$ be a Borel set such that $\mu(G \cdot B)=1$, let $(\bar{E}, \bar{g})$ be a fulfillment of $B$ with fulfillment function $f: \widetilde{E} \rightarrow G$, and let $\varphi$ be the fulfilled cocycle associated with $(\psi, B, \bar{E}, \bar{g})$. Suppose that both of the following conditions hold:

(a) $\psi$ is B-essentially continuous,

(b) $f$ is continuous.

Then $\varphi$ is essentially continuous.

Proof. Let $T \subset G \times X$ be a Borel set which witnesses the $B$-essential continuity of $\psi$. Now $\widetilde{E}$ is Borel, hence so is $Q(\widetilde{E})$. As $\mu(G \cdot B)=1$, by definition of fulfillment, $\mu(\widetilde{E})=1$; since $\mu$ is quasi-invariant, for all $g \in G, \mu\left((Q(\widetilde{E}))_{g}\right)=1$. Define a function $\theta: Q(\widetilde{E}) \rightarrow G \times X$ by

$$
\theta(g, x)=\left(f(g \cdot x) g(f(x))^{-1}, f(x) \cdot x\right) .
$$

Since $x$ and $g \cdot x$ are in $\widetilde{E}=\operatorname{dom}(f), \theta$ is well defined. The continuity of $f$ implies that $\theta$ is continuous. By definition of fulfilled cocycle (Definition 2.6.1), for all $(g, x) \in Q(\widetilde{E}), \varphi(g, x)=\psi(\theta(g, x))$. Now define

$$
S=\{(g, x) \in Q(\widetilde{E}): \theta(g, x) \in T\} .
$$

Clearly $S$ is a Borel subset of $G \times X$. Condition (b) of the definition of $B$-essential continuity states that $\psi\lceil T$ is continuous. Therefore, $\varphi\lceil S$ is continuous.

To show that $S$ witnesses the definition of essential continuity for $\varphi$, all that remains to be proved is that for all $g \in G$, for a.e. $x \in X,(g, x) \in S$. The proof of this is similar to the proofs of Lemmas 2.6.2 and 2.6.3. Suppose it is false: fix $k \in G$ and fix a Borel set $D \subset X$ such that $\mu(D)>0$ and for all $x \in D,(k, x) \notin S$. That is, for all $x \in\left[D \cap(Q(\widetilde{E}))_{k}\right], \theta(k, x) \notin T$. Let $\bar{E}=E_{0}, E_{1}, E_{2}, \ldots$ and 
$\bar{g}=g_{0}, g_{1}, g_{2}, \ldots$ Since $\mu\left((Q(\widetilde{E}))_{k}\right)=1$, there are natural numbers $m$ and $n$ such that if we set

$$
\widehat{D}=\left\{x \in D: x \in E_{m} \text { and } k \cdot x \in E_{n} \text { and }(k, x) \in Q(\widetilde{E})\right\},
$$

then $\mu(\widehat{D})>0$. By definition of fulfillment and of $\widehat{D}$, for all $x \in \widehat{D}: g_{m} \cdot x \in B$ and

$$
\left(g_{n} k g_{m}^{-1}\right) \cdot\left(g_{m} \cdot x\right)=g_{n} \cdot(k \cdot x) \in B
$$

Condition (a) of the definition of $B$-essentially continuous implies - taking $x$ to be $g_{m} \cdot x$ and $g$ to be $g_{n} k g_{m}^{-1}$ - that

$$
\mu\left(\left\{g_{m} \cdot x: x \in \widehat{D} \text { and }\left(g_{n} k g_{m}^{-1}, g_{m} \cdot x\right) \notin T\right\}\right)=0 .
$$

By quasi-invariance, for a.e. $x \in \widehat{D},\left(g_{n} k g_{m}^{-1}, g_{m} \cdot x\right) \in T$. For all $x \in \widehat{D},(k, x) \in$ $\operatorname{dom}(\theta)$ and

$$
\begin{aligned}
\theta(k, x) & =\left(f(k \cdot x) k(f(x))^{-1}, f(x) \cdot x\right) \\
& =\left(g_{n} k g_{m}^{-1}, g_{m} \cdot x\right) .
\end{aligned}
$$

So for a.e. $x \in \widehat{D}, \theta(k, x) \in T$. As $S=\theta^{-1}[T]$, for a.e. $x \in \widehat{D},(k, x) \in S$, a contradiction.

Lemma 2.6.6. Suppose that $X$ is a Polish $G$-space. Let $B \subset X$ be a Borel set such that $\mu(G \cdot B)=1$, and let $(\bar{E}, \bar{g})$ be a fulfilment of $B$ with fulfillment function $f: \widetilde{E} \rightarrow G$. Suppose that both of the following conditions hold:

(a) $\psi$ is B-essentially continuous,

(b) $f$ is continuous.

Then there exists an almost cocycle, $\varphi$, such that $\varphi$ is essentially continuous and $\varphi$ is cohomologous to $\psi$.

Proof. Let $\varphi$ be the fulfilled cocycle associated with $(\psi, B, \bar{E}, \bar{g})$. This result now follows from Lemmas 2.6.2, 2.6.3, and 2.6.5.

Lemma 2.6.7. Suppose that $\psi$ is nearly quintessentially continuous. There exists a compatible topology, $t$, for $X$, there exists a Borel set $B \subset X$ such that $\mu(G \cdot B)=1$, and there exists a fulfilment $(\bar{E}, \bar{g})$ of $B$ with fulfilment function $f: \widetilde{E} \rightarrow G$ such that both of the following conditions hold (with respect to the topology $t$ ):

(a) $\psi$ is B-essentially continuous,

(b) $f$ is continuous.

Proof. By definition of near quintessential continuity (Definition 1.4.3), there exists a compatible topology, $\tau$, for $X$ such that $\psi$ is nearly essentially continuous with respect to $\tau$. Let $\mathcal{F}$ be a maximal family of Borel subsets of $X$ satisfying the following four properties:

(i) For all $C \in \mathcal{F}, \mu(C)>0$.

(ii) For all $C, C^{\prime} \in \mathcal{F}$, if $C \neq C^{\prime}$, then $G \cdot C \cap G \cdot C^{\prime}=\emptyset$.

(iii) For all $C \in \mathcal{F}, \psi$ is $C$-essentially continuous with respect to $\tau$.

(iv) For all $C \in \mathcal{F}$, there exists a compatible topology, $\tau_{C}$, for $X$ and there exists a fulfillment of $C$ with fulfillment function $f_{C}: \widetilde{E}_{C} \rightarrow G$ such that $f_{C}$ is continuous with respect to $\tau_{C}$.

Claim. $\mu(\bigcup\{G \cdot C: C \in \mathcal{F}\})=1$. 
Proof of Claim. Let $P=X \backslash(\bigcup\{G \cdot C: C \in \mathcal{F}\})$. By (i) and (ii), $\mathcal{F}$ is countable, so $P$ is coanalytic, hence measurable. Now assume the Claim is false. Then $\mu(P)>0$. By definition of near essential continuity (Definition 1.4.1(b)), there exists a $\tau$ compact $K \subset X$ such that $\mu(K)>1-\mu(P)$ and such that $\psi$ is $K$-essentially continuous (see Definition 2.6.4) with respect to $\tau$. Let $A$ be a Borel subset of $K \cap P$ such that $\mu(A)>0$. Clearly $\psi$ is $D$-essentially continuous with respect to $\tau$ for all Borel subsets $D$ of $K$. Therefore, if $D$ is any Borel subset of $A$ of positive measure, and $D$ is added to $\mathcal{F}$, then (i)-(iii) still hold. By maximality, no Borel subset of $A$ of positive measure satisfies (iv). We now apply Theorem 1.2.12: there exists a Borel set $D \subset A$ such that $\mu(D)=\mu(A)>0$ and there exists a compatible topology, $\tau_{D}$, for $X$ and there exists a fulfillment of $D$ whose fulfillment function $f_{D}: \widetilde{E}_{D} \rightarrow G$ is continuous with respect to $\tau_{D}$. This contradiction proves the Claim.

Consider a fixed $C \in \mathcal{F}$. Both $G \cdot C$ and $G \cdot[\bigcup(\mathcal{F} \backslash\{C\})]$ are analytic sets, and, by (ii), they are disjoint. So by Lemma 2.1.16, there exists an invariant Borel set, $D_{C}$, such that $G \cdot C \subset D_{C}$ and $G \cdot[\bigcup(\mathcal{F} \backslash\{C\})] \cap D_{C}=\emptyset$. And by Theorem 2.1.17, there exists a compatible topology, $t_{C}$, for $X$ such that $D_{C}$ is $t_{C}$-open.

For each $C \in \mathcal{F}$, fix a $t_{C}$ as above, and fix $\tau_{C}$ and $f_{C}$ satisfying (iv). By Corollary 2.1.18, there exists a compatible topology, $t$, such that $t$ is finer than $\tau, t$ is finer than each $\tau_{C}$, and $t$ is also finer than each $t_{C}$. Let $B=\bigcup \mathcal{F}$. Clearly $B$ is Borel and, by the Claim, $\mu(G \cdot B)=1$. In light of (ii), we can take the "union" of the fulfillments of each $C$ in $\mathcal{F}$ to produce a fulfillment of $B$, with fulfillment function $f: \widetilde{E} \rightarrow G$ such that for all $C \in \mathcal{F}, f\left\lceil\widetilde{E}_{C}=f_{C}\right.$. It is now easy to deduce (a) from (iii) and (b) from (iv).

Proof of Theorem C. Lemmas 2.6.6 and 2.6.7.

2.7. Proofs of theorems about counterexamples. In $\S 2.7$, we prove the three theorems regarding counterexamples which were stated in $\S 1.3 . \mathrm{E}$ : Theorem 1.3.15 about the unsuitable counterexample and Theorems 1.3.18 and 1.3.19 about the suitable counterexample.

A. The unsuitable counterexample. Recall the Polish $S_{\infty}$-space $X_{0}$ of Definition 1.2.5 and the function $\sigma: X_{0} \rightarrow S_{\infty}$ of Definition 1.3.14.

Let $p \in X_{0} \subset 2^{\mathbb{N}}$ be the characteristic function of the odd numbers, and let

$$
K=\left\{g \in S_{\infty}: \text { for all } n \in \mathbb{N}, n \text { is odd iff } g(n) \text { is odd }\right\} .
$$

\section{Proposition 2.7.1. $\quad$ (a) $\sigma$ is continuous.}

(b) $K$ is the stabilizer subgroup of $p$.

(c) $\sigma(p)=e$.

(d) For all $x \in X_{0},(\sigma(x)) \cdot p=x$.

Definition 2.7.2. For any continuous homomorphism $\pi: K \rightarrow S_{\infty}$, define a function $\varphi^{\pi}: S_{\infty} \times X_{0} \rightarrow S_{\infty}$ by the formula

$$
\varphi^{\pi}(g, x)=\pi\left((\sigma(g \cdot x))^{-1} g \sigma(x)\right) .
$$

The construction of the $\varphi^{\pi}$ 's, above, is a special case of the construction in Example 1.3.2.

The next proposition follows easily from Proposition 2.7.1.

Proposition 2.7.3. For any continuous homomorphism $\pi: K \rightarrow S_{\infty}$, all four of the following facts are true. 
(a) $\varphi^{\pi}$ is a strict cocycle on the $S_{\infty}$-space $X_{0}$ with values in $S_{\infty}$.

(b) $\varphi^{\pi}$ is continuous.

(c) For all $g \in K, \varphi^{\pi}(g, p)=\pi(g)$.

(d) For all $x \in X_{0}, \varphi^{\pi}(\sigma(x), p)=e$.

Definition 2.7.4. Let $\varphi$ and $\psi$ be two strict cocycles on $X_{0}$ with values in $S_{\infty}$. We say that $\varphi$ is strictly cohomologous to $\psi$ if there exits a Borel-measurable function $F: X_{0} \rightarrow S_{\infty}$ such that for all $g \in S_{\infty}$, for all $x \in X_{0}$,

$$
\varphi(g, x)=F(g \cdot x) \psi(g, x)[F(x)]^{-1} .
$$

Lemma 2.7.5. Let $\psi: S_{\infty} \times X_{0} \rightarrow S_{\infty}$ be a Borel-measurable strict cocycle. There exists a continuous homomorphism $\pi: K \rightarrow S_{\infty}$ such that $\varphi^{\pi}$ is strictly cohomologous to $\psi$.

Proof. Let $\pi: K \rightarrow S_{\infty}$ be the Borel function $\pi(g)=\psi(g, p)$. By Proposition 2.7.1(b), $p=g \cdot p$; hence the cocycle identity implies that $\pi$ is a homomorphism. By Lemma 2.1.13, a Borel homomorphism is continuous.

Let $F: X_{0} \rightarrow S_{\infty}$ be the Borel-measurable function $F(x)=[\psi(\sigma(x), p)]^{-1}$. To complete the proof, we need only show that for all $g \in S_{\infty}$ and for all $x \in X_{0}$,

$$
\varphi^{\pi}(g, x)=F(g \cdot x) \psi(g, x)[F(x)]^{-1} .
$$

Fix $g$ and $x$.

Claim. Let $h=(\sigma(g \cdot x))^{-1} g \sigma(x)$.

(a) $\psi(h, p)=\varphi^{\pi}(h, p)$.

(b) $\psi(h, p)=[\psi(\sigma(g \cdot x), p)]^{-1} \psi(g, x) \psi(\sigma(x), p)$.

(c) $\varphi^{\pi}(h, p)=\left[\varphi^{\pi}(\sigma(g \cdot x), p)\right]^{-1} \varphi^{\pi}(g, x) \varphi^{\pi}(\sigma(x), p)$.

Proof of Claim. Using part (d) of Proposition 2.7.1, we see that

$$
\begin{aligned}
h \cdot p & =\left[[\sigma(g \cdot x)]^{-1} g \sigma(x)\right] \cdot p \\
& =\left[[\sigma(g \cdot x)]^{-1} g\right] \cdot[\sigma(x) \cdot p] \\
& =\left[[\sigma(g \cdot x)]^{-1} g\right] \cdot x \\
& =[\sigma(g \cdot x)]^{-1} \cdot(g \cdot x) \\
& =p .
\end{aligned}
$$

So by part (b) of that Proposition, $h \in K$. Hence by Proposition 2.7.3(c) and the definition of $\pi$,

$$
\varphi^{\pi}(h, p)=\pi(h)=\psi(h, p),
$$

which proves (a). Again using Proposition 2.7.1(d), as well as Proposition 2.1.22 (a)(ii) and the cocycle identity, we obtain the following equations:

$$
\begin{aligned}
\psi(h, p) & =\psi\left((\sigma(g \cdot x))^{-1} g \sigma(x), p\right) \\
& =\psi\left((\sigma(g \cdot x))^{-1}, g \cdot(\sigma(x) \cdot p)\right) \psi(g, \sigma(x) \cdot p) \psi(\sigma(x), p) \\
& =\psi\left((\sigma(g \cdot x))^{-1}, g \cdot x\right) \psi(g, x) \psi(\sigma(x), p) \\
& =\left[\psi\left(\sigma(g \cdot x),(\sigma(g \cdot x))^{-1} \cdot(g \cdot x)\right)\right]^{-1} \psi(g, x) \psi(\sigma(x), p) \\
& =[\psi(\sigma(g \cdot x), p)]^{-1} \psi(g, x) \psi(\sigma(x), p) .
\end{aligned}
$$

This proves (b). The proof of (c) is identical to the proof of (b). Thus the proof of the Claim is complete. 
Part (c) of the Claim plus Proposition 2.7.3(d) establish that $\varphi^{\pi}(h, p)=\varphi^{\pi}(g, x)$. Therefore, by parts (a) and (b) of the Claim,

$$
\begin{aligned}
\varphi^{\pi}(g, x) & =[\psi(\sigma(g \cdot x), p)]^{-1} \psi(g, x) \psi(\sigma(x), p) \\
& =F(g \cdot x) \psi(g, x)[F(x)]^{-1} .
\end{aligned}
$$

Definition 2.7.6. Let $Y_{0}=\left\{x \in X_{0}: x(0)=x(1)=0\right\}$. Let $g_{0}$ denote the element of $S_{\infty}$ which transposes 0 and 1 and fixes all other elements of $\mathbb{N}$. For any Borel strict cocycle $\psi: S_{\infty} \times X_{0} \rightarrow S_{\infty}$, let

$$
Z(\psi)=\left\{x \in Y_{0}: \psi\left(g_{0}, x\right)=e\right\} .
$$

Lemma 2.7.7. $\quad$ (a) $Y_{0}$ is clopen and nonempty.

(b) For any Borel-measurable strict cocycle $\psi: S_{\infty} \times X_{0} \rightarrow S_{\infty}, Z(\psi)$ is closed.

Proof. (b) By Lemma 2.7.5, there exists a continuous homomorphism $\pi: K \rightarrow S_{\infty}$ and there exists a Borel function $F: X_{0} \rightarrow S_{\infty}$ such that $F$ witnesses that $\varphi^{\pi}$ is strictly cohomologous to $\psi$. For $x \in Y_{0}$,

$$
\begin{aligned}
\varphi^{\pi}\left(g_{0}, x\right) & =F\left(g_{0} \cdot x\right) \psi\left(g_{0}, x\right)[F(x)]^{-1} \\
& =F(x) \psi\left(g_{0}, x\right)[F(x)]^{-1} .
\end{aligned}
$$

So $Z(\psi)=Z\left(\varphi^{\pi}\right)$. This lemma thus follows from part (b) of Proposition 2.7.3.

Proposition 2.7.8. Let $\nu$ be any quasi-invariant Borel probability measure on the $S_{\infty}$-space $X_{0}$. Let $Y \subset X_{0}$ be any nonempty open set. Then $\nu(Y)>0$.

Recall the two strict cocycles of Definition 1.3.14: the trivial cocycle, $\varphi_{t}(g, x)=$ $e$, and the interesting cocycle, $\varphi_{i}(g, x)=(\sigma(g \cdot x))^{-1} g \sigma(x)$.

Proof of Theorem 1.3.15. Let $\mu$ be a quasi-invariant Borel probability measure on $X_{0}$ and let $Q \subset X_{0}$ be a $\mu$-almost invariant Borel set such that $0<\mu(Q)<1$. Let $\tilde{\varphi}: S_{\infty} \times X_{0} \rightarrow S_{\infty}$ be the function

$$
\tilde{\varphi}(g, x)=\left\{\begin{array}{lll}
\varphi_{t}(g, x), & \text { if } & x \in Q, \\
\varphi_{i}(g, x), & \text { if } & x \notin Q .
\end{array}\right.
$$

It is obvious that $\tilde{\varphi}$ is a Borel-measurable almost cocycle. We must prove that $\tilde{\varphi}$ is not equivalent (with respect to $\mu$ ) to any Borel-measurable strict cocycle.

Assume, towards a contradiction, that $\tilde{\varphi}$ is equivalent (with respect to $\mu$ ) to a Borel-measurable strict cocycle - call it $\psi$. Let $\mu_{1}=\mu\lceil Q$, normalized, and let $\mu_{2}=\mu\left\lceil\left(X_{0} \backslash Q\right)\right.$, normalized. As $Q$ is $\mu$-almost invariant, $\mu_{1}$ and $\mu_{2}$ are both quasiinvariant measures. Note that $\tilde{\varphi}$ is equivalent to $\psi$ with respect to both $\mu_{1}$ and $\mu_{2}$.

For all $x \in Q, \tilde{\varphi}\left(g_{0}, x\right)=\varphi_{t}\left(g_{0}, x\right)=e$. So for $\mu_{1}$-a.e. $x \in X_{0}, \psi\left(g_{0}, x\right)=e$. By Lemma 2.7.7 and Proposition 2.7.8, $Z(\psi)=Y_{0}$.

For all $x \in Y_{0} \backslash Q$,

$$
\tilde{\varphi}\left(g_{0}, x\right)=\varphi_{i}\left(g_{0}, x\right)=\left(\sigma\left(g_{0} \cdot x\right)\right)^{-1} g_{0} \sigma(x)=(\sigma(x))^{-1} g_{0} \sigma(x) .
$$

As $g_{0}$ is not the group identity, none of its conjugates are. Thus, for all $x \in$ $Y_{0} \backslash Q, \tilde{\varphi}\left(g_{0}, x\right) \neq e$. So for $\mu_{2}$-a.e. $x \in Y_{0}, \psi\left(g_{0}, x\right) \neq e$. Therefore, $\mu_{2}(Z(\psi))=0$.

We have now shown that $\mu_{2}\left(Y_{0}\right)=0$. By Proposition 2.7.8, $Y_{0}$ cannot be a nonempty open set. This contradicts Lemma 2.7.7(a). 
B. The suitable counterexample. Throughout $\S 2.7$.B, we work with the Polish group $G^{0}$ of Definition 1.3.16 and the measured Polish $G^{0}$-space $(\mathbb{T}, \lambda)$, where $\mathbb{T}$ is the unit circle, $\lambda$ is normalized Lebesgue measure, and $G^{0}$ acts on $\mathbb{T}$ by evaluation.

Definition 2.7.9. Let $R$ denote the group of rotations of $\mathbb{T}$. We also let $\mathbb{T}$ denote the Polish $R$-space (under the evaluation action).

Of course, $R$ is a closed subgroup of $G^{0}$, hence Polish, and $(\mathbb{T}, \lambda)$ is a measured Polish $R$-space.

Lemma 2.7.10. $\lambda$ is a suitable measure on the $R$-space $\mathbb{T}$.

Proof. Theorem 1.2.9.

Definition 2.7.11. Let $A$ and $B$ be Borel subsets of $\mathbb{T}$, and let $H$ be a closed subgroup of $G^{0}$, acting on $\mathbb{T}$ by evaluation.

(a) We say that the triple $(A, B, H)$ satisfies condition 1 if the following holds. Let $M \subset H$ be any open neighborhood of $H$ containing $e$. There exists a $g \in M$ such that $\lambda(\{x \in A: g \cdot x \in B\})>0$.

(b) We say that the triple $(A, B, H)$ satisfies condition 2 if there exist Borel sets $A^{\prime} \subset A$ and $B^{\prime} \subset B$ with $\lambda\left(A \backslash A^{\prime}\right)=\lambda\left(B \backslash B^{\prime}\right)=0$ and there exists an open neighborhood $N$ of $H$ containing $e$ such that $\left(N \cdot A^{\prime}\right) \cap B^{\prime}=\emptyset$.

Lemma 2.7.12. Let $A$ and $B$ be Borel subsets of $\mathbb{T}$.

(a) If $(A, B, R)$ satisfies condition 1 , then so does $\left(A, B, G^{0}\right)$.

(b) If $(A, B, R)$ satisfies condition 2 , then so does $\left(A, B, G^{0}\right)$.

Proof. (a) Obvious.

(b) Let $A^{\prime} \subset A$ and $B^{\prime} \subset B$ witness that $(A, B, R)$ satisfies condition 2. Then there exists an open neighborhood $N^{*}$ of $R$ containing the group identity such that $\left(N^{*} \cdot A^{\prime}\right) \cap B^{\prime}=\emptyset$. By definition of $R$ and of the $R$-space, this means that there is an $\varepsilon>0$ such that for all $x \in A^{\prime}$, for all $y \in B^{\prime}, \delta(x, y)>\varepsilon$ (where $\delta$ is the usual metric on $\mathbb{T}$ ). Let

$$
N=\left\{h \in G^{0}: \delta^{1}(e, h)<\varepsilon\right\} .
$$

By definition of the topology of $G^{0}$ (Definition 1.3.16), $N$ is an open neighborhood of $G^{0}$ containing $e$. Clearly $\left(N \cdot A^{\prime}\right) \cap B^{\prime}=\emptyset$.

Proof of Theorem 1.3.18. We must prove that $\lambda$ is a suitable measure on the $G^{0}$ space $\mathbb{T}$. Suitable means, by Definition 1.2.7, that for all Borel $A, B \subset X$, either condition 1 or condition 2 is satisfied. Therefore, this theorem follows from Lemmas 2.7.10 and 2.7.12.

Definition 2.7.13. Let $y \in \mathbb{T}$, and let $n \geq 3$ be an integer.

(a) $I_{n}^{y}$ denotes the open interval of $\mathbb{T}$ centered at $y$ with measure $1 / n$.

(b) $J_{n}^{y}$ denotes the open interval of $\mathbb{T}$ centered at $y$ with measure $2 / n$.

(c) Let $g_{n}^{y}$ denote the continuous function from $\mathbb{T}$ onto $\mathbb{T}$ with the following properties: $g_{n}^{y}$ is orientation-preserving; $g_{n}^{y}\left[I_{n}^{y}\right]=J_{n}^{y}$ and $g_{n}^{y}\left\lceil I_{n}^{y}\right.$ has a constant rate of magnification of $2 ; g_{n}^{y}\left[\mathbb{T} \backslash I_{n}^{y}\right]=\mathbb{T} \backslash J_{n}^{y}$ and $g_{n}^{y}\left\lceil\left(\mathbb{T} \backslash I_{n}^{y}\right)\right.$ has a constant rate of contraction.

Lemma 2.7.14. For all $y \in \mathbb{T}$, the following three properties hold:

(a) For all $n \geq 3, g_{n}^{y} \in G^{0}$. 
(b) For all $n \geq 3$, for $\lambda$-a.e. $x \in I_{n}^{y}$,

$$
\frac{d\left(\left(g_{n}^{y}\right)^{-1} \cdot \lambda\right)}{d \lambda}(x)=2 .
$$

(c) The sequence $g_{n}^{y}$ converges to $e$ (in the topology of $G^{0}$ ) as $n \rightarrow \infty$.

Proof. It is obvious that $g_{n}^{y}$ is an orientation-preserving homeomorphism of $\mathbb{T}$ and that it and its inverse preserve measure 0 sets. So $g_{n}^{y} \in G^{0}$, and (a) holds.

It is also obvious what the Radon-Nikodým derivatives

$$
\frac{d\left(g_{n}^{y} \cdot \lambda\right)}{d \lambda} \text { and } \frac{d\left(\left(g_{n}^{y}\right)^{-1} \cdot \lambda\right)}{d \lambda}
$$

are. In particular, (b) holds, and for $\lambda$-a.e. $x \in \mathbb{T}$,

$$
\begin{aligned}
& \text { (i) } \frac{d\left(g_{n}^{y} \cdot \lambda\right)}{d \lambda}\left\lceil J_{n}^{y}=\frac{1}{2},\right. \\
& \text { (ii) } \frac{d\left(g_{n}^{y} \cdot \lambda\right)}{d \lambda}\left\lceil\left(\mathbb{T} \backslash J_{n}^{y}\right)=\frac{1-\frac{1}{n}}{1-\frac{2}{n}} .\right.
\end{aligned}
$$

We now prove (c). Recall the functions $\delta^{1}$ and $\delta^{2}$ used in defining the topology of $G^{0}$ in Definition 1.3.16. Note that in formula (ii) of (2.7.15), the right-hand side of the equation converges to 1 as $n \rightarrow \infty$. So (2.7.15) implies first, that the sequence $d\left(g_{n}^{y} \cdot \lambda\right) / d \lambda$ is uniformly bounded $\lambda$-almost everywhere, and second, that this sequence of functions converges to $1 \lambda$-almost everywhere. As $d(e \cdot \lambda) / d \lambda$ is $1 \lambda$-almost everywhere, $\delta^{2}\left(g_{n}^{y}, e\right)$ converges to 0 . Now $g_{n}^{y}$ converges uniformly to the identity function, so $\delta^{1}\left(g_{n}^{y}, e\right)$ converges to 0 . By definition of the topology of $G^{0}, g_{n}^{y}$ converges to $e$ in that topology.

Proof of Theorem 1.3.19. Let $\varphi: G^{0} \times \mathbb{T} \rightarrow \mathbb{R}^{*}$ be the Radon-Nikodým Cocycle. We must prove that $\varphi$ is not equivalent to any Borel-measurable strict cocycle.

Suppose that is false: $\varphi$ is equivalent to a Borel-measurable strict cocycle. The Main Theorem (Theorem 1.4.7) then tells us that $\varphi$ is nearly quintessentially continuous.

By Proposition 2.1.22(b)(i), for $\lambda$-a.e. $x \in \mathbb{T}, \varphi(e, x)$ is the group identity of $\mathbb{R}^{*}$, which is an element of the open neighborhood $(0,2)$ of $\mathbb{R}^{*}$. By definition of the Radon-Nikodým Cocycle (see Example 1.3.5), for all $g \in G^{0}$, for $\lambda$-a.e. $x \in \mathbb{T}$,

$$
\varphi(g, x)=\frac{d\left(g^{-1} \cdot \lambda\right)}{d \lambda}(x) .
$$

So it follows from the definition of nearly quintessentially continuous (see Definitions 1.4.1(b) and 1.4.3) that there exists a Borel set $A \subset \mathbb{T}$ with $\lambda(A)>0$ and there exists an open neighborhood $M$ of $G^{0}$ with $e \in M$ such that

$$
\begin{aligned}
& \text { for all } g \in M \text {, for } \lambda \text {-a.e. } x \in \mathbb{T} \text {, if both } \\
& \qquad x \text { and } g \cdot x \text { are in } A \text {, then } \frac{d\left(g^{-1} \cdot \lambda\right)}{d \lambda}(x) \in(0,2) .
\end{aligned}
$$

As $\lambda(A)>0$, the Lebesgue Density Theorem 2.1.5 gives us a point $y \in \mathbb{T}$ such that $A$ has density 1 at $y$. This implies that for sufficiently large $n$,

$$
\begin{aligned}
\text { (i) } & \frac{\lambda\left(A \cap I_{n}^{y}\right)}{\lambda\left(I_{n}^{y}\right)}>\frac{1}{2}, \\
\text { (ii) } & \frac{\lambda\left(A \cap J_{n}^{y}\right)}{\lambda\left(J_{n}^{y}\right)}>\frac{1}{2} .
\end{aligned}
$$


Using part (c) of Lemma 2.7.14, we can fix an $n$ large enough that $g_{n}^{y} \in M$ and both parts of (2.7.17) hold. Let $g=g_{n}^{y}$.

By part (b) of Lemma 2.7.14, for $\lambda$-a.e. $x \in I_{n}^{y}, \frac{d\left(g^{-1} \cdot \lambda\right)}{d \lambda}(x)=2$. So $(2.7 .16)$ implies that for $\lambda$-a.e. $x \in I_{n}^{y}$, either $x$ or $g \cdot x$ is not in $A$. This fact, together with (2.7.17)(i), establishes that

$$
\lambda\left(\left\{x \in I_{n}^{y}: g \cdot x \in A\right\}\right)<\frac{1}{2} \cdot \lambda\left(I_{n}^{y}\right) .
$$

Using the definitions of $I_{n}^{y}, J_{n}^{y}$, and $g_{n}^{y}$, we now see that

$$
\begin{aligned}
\lambda\left(A \cap J_{n}^{y}\right) & =\lambda\left(\left\{g \cdot x \in J_{n}^{y}: g \cdot x \in A\right\}\right) \\
& =2 \cdot \lambda\left(\left\{x \in I_{n}^{y}: g \cdot x \in A\right\}\right) \\
& <2 \cdot\left(\frac{1}{2} \cdot \lambda\left(I_{n}^{y}\right)\right) \\
& =\lambda\left(I_{n}^{y}\right) \\
& =\frac{1}{2} \cdot \lambda\left(J_{n}^{y}\right) .
\end{aligned}
$$

This contradicts (2.7.17)(ii).

\section{REFERENCES}

[1] M. Balcerzak, A classification of $\sigma$-ideals in Polish groups, Demonstratio Mathematica 20(1987), 77-88. MR941406 (89e:54076)

[2] H. Becker, Polish group actions: dichotomies and generalized elementary embeddings, Journal of the American Mathematical Society 11(1998), 397-449. MR1478843 (99g:03051)

[3] H. Becker and A.S. Kechris, The Descriptive Set Theory of Polish Group Actions, Cambridge University Press, 1996. MR.1425877 (98d:54068)

[4] R.C. Fabec, Fundamentals of Infinite Dimensional Representation Theory, Chapman and Hall/CRC, 2000. MR:1775825(2001m:22001)

[5] F. Fidaleo, Remarks on the imprimitivity theorem for nonlocally compact Polish groups, Infinite Dimensional Analysis, Quantum Probability and Related Topics 3(2000), 247-262. MR:1812700 (2002e:22001)

[6] W. Herer and J.P.R. Christensen, On the existence of pathological submeasures and the construction of exotic topological groups, Math. Ann. 213(1975), 203-210. MR0412369 (54:495)

[7] A.S. Kechris, Classical Descriptive Set Theory, Springer-Verlag, $1995 . \quad$ MR1321597 (96e:03057)

[8] G.W. Mackey, Ergodic theory and virtual groups, Math. Ann. 166(1966), 187-207. MR0201562 (34:1444)

[9] G.W. Mackey, Unitary Group Representations in Physics, Probability and Number Theory, Addison-Wesley, 1989. MR.1043174 (90m:22002)

[10] D. Montgomery and L. Zippin, Topological Transformation Groups, Interscience Publishers, 1955. MR0073104 (17:383b)

[11] Y.N. Moschovakis, Descriptive Set Theory, North-Holland, 1980. MR561709 (82e:03002)

[12] J.C. Oxtoby, Measure and Category, second edition, Springer-Verlag, 1980. MR584443 (81j:28003)

[13] I. Recław and P. Zakrzewski, Fubini properties of ideals, Real Analysis Exchange 25(1999/2000), 565-578. MR1778511 (2001e:03086)

[14] S. Solecki, Polish group topologies, in Sets and Proofs, (S.B. Cooper and J.K. Truss, Eds.), Cambridge University Press, 1999, 339-364. MR 1720580 (2000j:54045)

[15] V.S. Varadarajan, Geometry of Quantum Theory, second edition, Springer-Verlag, 1985. MR805158(87a:81009)

[16] R. Vaught, Invariant sets in topology and logic, Fund. Math. 82(1974), 269-294. MR0363912 (51:167) 
[17] A.M. Vershik, Kolmogorov's example (a survey of actions of infinite-dimensional groups with an invariant probability measure), Theory of Probability and its Applications 48(2004), 373378. MR2015459 (2004m:37009)

PMB 128, 4840 Forest Dr., Ste. 6-B, Columbia, South Carolina 29206-4810

E-mail address: hsbecker@hotmail.com 